\title{
Nonlinear optics and saturation behavior of quantum dot samples under continuous wave driving
}

T. Ackemann, A. Tierno, R. Kuszelewicz, S. Barbay, M. Brambilla, C. G. Leburn, and C. T. A. Brown

\begin{abstract}
The nonlinear optical response of self-assembled quantum dots is relevant to the application of quantum dot based devices in nonlinear optics, all-optical switching, slow light and self-organization. Theoretical investigations are based on numerical simulations of a spatially and spectrally resolved rate equation model, which takes into account the strong coupling of the quantum dots to the carrier reservoir created by the wetting layer states. The complex dielectric susceptibility of the ground state is obtained. The saturation is shown to follow a behavior in between the one for a dominantly homogeneously and inhomogeneously broadened medium. Approaches to extract the nonlinear refractive index change by fringe shifts in a cavity or self-lensing are discussed. Experimental work on saturation characteristic of $\mathrm{InGa} / \mathrm{GaAs}$ quantum dots close to the telecommunication O-band (1.24-1.28 $\mu \mathrm{m})$ and of InAlAs/GaAlAs quantum dots at $780 \mathrm{~nm}$ is described and the first demonstra-
\end{abstract}

T. Ackemann

SUPA and Department of Physics, University of Strathclyde, Glasgow G4 0NG, Scotland, UK. e-mail: thorsten.ackemann@strath.ac.uk

\section{A. Tierno}

Université de Nice Sophia Antipolis, Institut Non-Linéaire de Nice, UMR 6618, 06560 Valbonne, France. e-mail: alessio.tierno@inln.cnrs.fr

R. Kuszelewicz, S. Barbay

Laboratoire de Photonique et de Nanostructures, CNRS, Route de Nozay, 91460 Marcoussis, France. e-mail: robert.kuszelewicz@lpn.cnrs.fr, sylvain.barbay@lpn.cnrs.fr

M. Brambilla

CNISM e Dipartimento Interateneo di Fisica, via Amendola 173, 70126 Bari, Italy. e-mail: brambilla@fisica.uniba.it

C. G. Leburn

SUPA and School of Engineering and Physical Sciences, Heriot-Watt University, Edinburgh EH14 4AS, Scotland, UK. e-mail: C.G.Leburn@hw.ac.uk

C. T. A. Brown

SUPA and School of Physics and Astronomy, University of St. Andrews, St. Andrews, KY16 9SS, Scotland, UK. e-mail: ctab@st-andrews.ac.uk 
tion of the $\mathrm{cw}$ saturation of absorption in room temperature quantum dot samples is discussed in detail.

\section{Introduction}

As witnessed by the contributions in this book, semiconductor quantum dots (QD) are finding considerable interest for laser, amplifier and quantum information devices. The 3D quantum confinement leads to a 'quasi-atomic' behavior with a delta function-like density of states resulting, from the applications point of view, in many realized or anticipated benefits such as low threshold currents, low temperature sensitivity and low phase-amplitude coupling.

From the fundamental point of view, it seems interesting to revisit linear and nonlinear effects known for atoms - or their archetypical abstraction, the 'two-level' atom, in QD 'artificial' atoms. In contrast to real atoms, QD size, density and material composition can be used to tailor emission wavelengths and other characteristics. The analogy between QD and atoms was most explored for single dots due to their relevance for quantum information devices, e.g. [1, 2, 3]. Saturation behavior typical for two-level atoms is reported in these Refs. However, in order to take advantage of long dephasing times cryogenic temperatures are required. Moving over to ensembles of QD, but still at cryogenic temperatures, self-induced transparency was demonstrated as a specific phenomenon in nonlinear beam propagation in twolevel systems [4], and spectral hole burning in [5] mimicking the corresponding effect in Doppler broadened atomic systems.

At room temperature, work on nonlinear optical properties focused on the gain regime due to the relevance for semiconductor optical amplifiers (SOAs), most often under pulsed excitation $[6,7,8,9,10,11,12]$, but also under $\mathrm{cw}$ conditions $[6,9,13$, $14,15,16]$. In contrast, studies under absorptive conditions seem to be limited either to pulsed excitation $[7,8,17,18,19]$, motivated by applications as semiconductor saturable absorption mirrors (SESAMs), or to collodial ensembles [20, 21].

However, absorptive and refractive index nonlinearities of ensembles of QD without inversion might be useful for photonic devices displaying optical bistability, optical pattern formation, spatial solitons or slow light [22, 23, 24, 25, 26]. In particular, many of the current authors have an interest in spatial cavity solitons (CS), i.e. self-confined solitary states in cw driven nonlinear cavities (see [27, 28, 29] for a review). CS are bistable and have 'mobility', i.e. are easily being steered around within the device aperture. Hence, they are attractive for all-optical processing applications [30, 31, 32]. Though CS should exist for self-focusing, absorptive and even self-defocusing situations, the self-focusing case appears to be the most robust by far [33, 31]. Hence the quasi-atomic character of the QD susceptibility is beneficial because a self-focusing or self-defocusing situation can be realized by simply changing the detuning whereas a bulk or quantum-well semiconductor is always self-defocusing under absorptive and self-focusing under amplifying condi- 
tions. Obviously, this enhanced flexibility of QD might be not only beneficial for solitons but for nonlinear optics in general.

Moreover, characterizing the nonlinear index response to external optical driving provides also an alternative approach to the important problem of characterizing phase-amplitude coupling in QD. Due to their symmetric, atom-like gain spectrum, 'ideal' QD should have zero phase-amplitude coupling or linewidth enhancement factor (or $\alpha$-factor [34]) at gain maximum and hence a reduced tendency to instabilities compared to quantum well and bulk devices. Indeed, a reduced $\alpha$-factor and a reduced tendency to beam filamentation was observed in many QD samples under some operating conditions [35, 36, 37, 38], but also fairly strong phase-amplitude coupling under different operating conditions $[38,39,40]$. Characterizing nonlinear phase shifts and/or the resulting self-lensing effects would give a direct indication of the tendency of the system to filamentation and help to identify the appropriate operation conditions for applications demanding low (lasers, amplifiers, absorptive nonlinearities) and high (dispersive optical nonlinearities) phase-amplitude coupling.

Hence, we are addressing in this chapter the room temperature, cw nonlinear optics of QDs. We review and give further details on a series of papers on modeling the complex nonlinear susceptibility of QD and more recently on the experimental observation of absorptive nonlinearities [22, 23, 24, 25, 41, 42]. Sec. 2 provides the theoretical framework. Due to the significant coupling of QDs to the semiconductor matrix they are incorporated in, QDs are more complex than 'simple' atoms and we are adopting a model including QD and wetting layer (WL) dynamics with the basic coupling mechanisms using phenomenological rate constants. Another complication comes from the fact that QD spectra correspond to a 'Voigt'-profile where neither the homogeneous nor the inhomogeneous broadening is strongly dominant. We derive the nonlinear dielectric susceptibility of the QD ground state (ES) and how it couples to nonlinear propagation and cavity equations. Predictions for the saturation behavior, nonlinear phase shifts, self-lensing, $\alpha$-factor and optical bistability are discussed. In Sec. 3, we investigate the saturation of InAs QD in a GaAs matrix emitting close to the telecommunication O-band. We demonstrate cw saturation of absorption and gain using a tunable Chromium forsterite laser as a pump laser. InAs/GaAs is maybe the most established QD material system due to the fact that it provides good quality lasing devices in the 1100-1250 nm wavelength range, where quantum well based devices are highly strained, and allows devices to reach the $1.3 \mu \mathrm{m}$ telecommunication band using the beneficial GaAs material system. This maturity and applicability at telecommunication wavelengths, where all-optical processing applications should happen, makes InAs QD attractive for these studies. They have, however, also a drawback, which is that their internal structures is not really simple but permits one or two excited QD states (ES) in addition to the GS. Hence, we are investigating in Sec. 4 InAlAs QD in an AlGaAs matrix operating around $780 \mathrm{~nm}$. These QD are highly confined dots and a possible ES should be very close in energy to the WL, much closer than the energy difference between ES and GS. Hence, treating WL and ES as a common reservoir is expected to be a better approximation than in InAs QD. They can be also conveniently driven with a titanium-sapphire laser. We demonstrate saturation of absorption under $\mathrm{cw}$ driv- 
ing also for these. Sec. 5 provides a brief summary of the issues discussed and an outlook on improving the nonlinear figure of merit of devices.

\section{Modeling and simulation results}

\subsection{The Model}

The modeling of semiconductor QD requires a number of assumptions, according to a variety of preconditions: in the first instance, the growth and sample characteristics, as well as the current and/or optical injection conditions. The most appealing feature of QD being their quasi-atomic susceptibility, one aims to have a clear set of discrete states from quantum confinement, the best separated as possible from the carriers states defined in the wetting layer (WL) which is generally considered as a quantum well and the barrier substrate where the carriers are injected via electric contacts. The description of the QD states depends, in turn, on the sample inasmuch as, e.g., Stranski-Krastanow (SK) growth induces an inhomogeneous broadening of the energy dot states, while a submonolayer deposition yields a larger homogeneity of the dot size [36]. In this work, we will consider a system of small and/or shallow confined QD so that either there is only one electron and one hole bound states, or in case other discrete states exists, these are well separated from the inhomogeneously broadened fundamental transition. The InAlAs/AlGaAs QD discussed in Sec. 4 are an example for the latter, whereas in the InAs/GaAs QD discussed in Sec. 3 several excited QD states (ES) roughly equally spaced between WL and GS exist. In our treatment, the carriers in the ES [14, 16] are not explicitly taken into account but it is assumed that WL and excited states constitute a common reservoir for the QD ground state population [13]. Due to the large separation between the lifetimes of the carriers in the QD ground state (100 ps to $2 \mathrm{~ns}$ ) and the fast coupling between the other states to the ground state (100 fs to some ps) $[16,9]$ the details of this coupling are not very important for the properties of the $\mathrm{cw}$ state, if probing and pumping are done at the same frequency. Hence, we will use 'QD population' synonymous to 'QD ground state population' and 'WL layer population' synonymous to 'WL and excited QD state population'.

The WL continuum states are expected not to significantly contribute to the optical susceptibility in the vicinity of the fundamental transition. Hence, we are only taking into account the contribution of the GS transition to the susceptibility. However, the electromagnetic field couples to the GS transition of all the QDs, with varying strength, which reflects the inhomogeneous broadening. In addition, the optical response is affected indirectly by the WL states because they couple to the GS via the carrier dynamics and a change of carrier density in the GS changes the susceptibility. The optical response of our model is thus characterized by the nonlinear coupling of the QD fundamental transition to the WL. The presence of other discrete 
and continuous states is taken into account via the relaxation mechanism between the GS states and the outside world (WL).

In the model we describe the articulate and still partially unclear relaxation mechanisms of QD ensembles through phenomenological transition and escape/capture rates for intra-dot decays/recombinations and QD-WL carrier relaxations. The spin dynamics is neglected since the spin memory is lost very fast at room temperature [43, 44].

The coupling mechanisms between the WL and the QD considered are the carrier escape and capture by the dot via thermo-activation through emission or absorption of lattice phonons and Auger processes at first order in the WL populations, which include Pauli blocking effects, under the assumption that the sample is passive or weakly pumped [45].

\subsubsection{Carrier dynamics and dielectric susceptibility}

Following [23], we derive the equations for the expectation values of the number of particles operator for the electrons and holes and for the corresponding polarization $p$ :

$$
\begin{gathered}
\frac{d n^{e, h}}{d t}=-\gamma_{n r} n^{e, h}-\Gamma_{s p} n^{e} n^{h}+\frac{i}{\hbar}\left(\mu p^{*}-\mu^{*} p\right) \mathrm{E}+\left.\frac{d n^{e, h}}{d t}\right|_{Q D-W L}+\left.\frac{d n^{e, h}}{d t}\right|_{Q D-W L} ^{\text {Auger }}, \\
\frac{d p}{d t}=-\left(i \omega_{a}+\gamma_{p}\right) p-\frac{i}{\hbar}\left[n^{e}+n^{h}-1\right] d \mathrm{E}
\end{gathered}
$$

where $\gamma_{n r}$ is the non radiative recombination rate, $\Gamma_{s p}$ the bimolecular coefficient for spontaneous recombination, $\omega_{a}$ the electron-hole recombination pulsation, $\gamma_{p}$ the polarization damping, $\mathrm{E}=E \exp \left(-i \omega_{0} t\right)+$ c.c. is the electric field and $\mu$ the dipole transition matrix element.

If we consider the level degeneracy $\Pi=2$ for the two opposite spins, we can introduce the QD population $n_{Q D}^{e, h}=\Pi n^{e, h}$, the total polarization $p_{Q D}=\Pi p$ and assume a real dipole moment $\mu$. In the rotating wave approximation and introducing $p_{Q D}=P_{Q D} \exp \left(-i \omega_{0} t\right)+c . c$. , Eqs. $(1,2)$ become

$$
\begin{gathered}
\frac{d P_{Q D}}{d t}=-\gamma_{p}(i \Delta+1) P_{Q D}-\frac{i \mu E}{\hbar}\left[n_{Q D}^{e}+n_{Q D}^{h}-\Pi\right] \\
\frac{d n_{Q D}^{e, h}}{d t}=-\gamma_{n r} n_{Q D}^{e, h}-\frac{\Gamma_{s p}}{2} n_{Q D}^{e} n_{Q D}^{h}+\frac{i \mu}{\hbar}\left(E P_{Q D}^{*}-E^{*} P_{Q D}\right)+ \\
\left.\frac{\partial n_{Q D}^{e, h}}{\partial t}\right|_{Q D-W L}+\left.\frac{\partial n_{Q D}^{e, h}}{\partial t}\right|_{Q D-W L} ^{A u g e r}
\end{gathered}
$$


where we have introduced the detuning from a single dot resonance $\Delta=\left(\omega_{a}-\right.$ $\left.\omega_{0}\right) / \gamma_{p}$

The first QD-WL relaxation term describes the thermo-activated processes. For the sake of simplicity we ignore the possible non local interaction between the QD and the WL, drop the subscript QD in the variable names and write

$$
\left.\frac{\partial n^{e, h}}{\partial t}\right|_{Q D-W L}=-\gamma_{e s c}^{e, h} n^{e, h}+\sigma_{c a p}^{e, h} N_{W L}^{e, h}\left[\Pi-n^{e, h}\right],
$$

where $N_{W L}^{e, h}$ is the surface density carrier population in the WL. $\gamma_{e s c}^{e, h}$ and $\sigma_{c a p}^{e, h}$ are respectively the escape rate from the QD and the capture rate cross-section into the QD. The second QD-WL relaxation terms describe the Auger processes [45]. For a low WL carrier density, we retain only those terms that are in first order in $N_{W L}^{e, h}$ :

$$
\left.\frac{\partial n^{e}}{\partial t}\right|_{Q D-W L} ^{\text {Auger }}=-B_{h e} N_{W L}^{h} n^{e}\left[\Pi-n^{h}\right]+B_{e h} N_{W L}^{e} n^{h}\left[\Pi-n^{e}\right] .
$$

The first term describes the excitation of an electron to the WL via the interaction of a hole in the WL and in the QD, and the second term is a symmetric process that describes the capture of a WL electron in the QD via the interaction of a WL and a QD hole. For the sake of conciseness, we will refer to the symmetric process in the following by "sym." in the equations. $B_{h e, e h}$ has the units of a cross-sectional rate (area/time). A similar term exists for the holes:

$$
\left.\frac{\partial n^{h}}{\partial t}\right|_{Q D-W L} ^{\text {Auger }}=-\left.\frac{\partial n^{e}}{\partial t}\right|_{Q D-W L} ^{\text {Auger }}
$$

The QD polarization $P_{Q D}$ can be adiabatically eliminated due to the fast polarization decay time $\gamma_{p}^{-1}$ with respect to the other ones so that its steady state values is:

$$
P_{Q D}=-\frac{i \mu}{\hbar \gamma_{p}}\left(\frac{1-i \Delta}{1+\Delta^{2}}\right)\left[n^{e}+n^{h}-\Pi\right] E,
$$

and can be substituted into Eq. (4) to obtain

$$
\begin{aligned}
\frac{d n_{Q D}^{e, h}}{d t}=-\gamma_{n r} n_{Q D}^{e, h}- & \frac{\Gamma_{s p}}{2} n_{Q D}^{e} n_{Q D}^{h}- \\
& \frac{2 \mu^{2}}{\hbar^{2} \gamma_{p}}\left(\frac{1}{1+\Delta^{2}}\right)\left[n^{e}+n^{h}-\Pi\right]|\mathbf{E}|^{2}+ \\
& \left.\frac{\partial n_{Q D}^{e, h}}{\partial t}\right|_{Q D-W L}+\left.\frac{\partial n_{Q D}^{e, h}}{\partial t}\right|_{Q D-W L} ^{\text {Auger }}
\end{aligned}
$$

The rate equation for the WL carrier population is given by 


$$
\begin{aligned}
\frac{\partial N_{W L}^{e, h}}{\partial t} & =\Lambda-\gamma_{n r}^{W L} N_{W L}^{e, h}+\left.\frac{\partial N_{W L}^{e, h}}{\partial t}\right|_{Q D-W L} \\
& +\left.\frac{\partial N_{W L}^{e, h}}{\partial t}\right|_{Q D-W L} ^{A u g e r}+D \nabla_{\perp}^{2} N_{W L}^{e, h},
\end{aligned}
$$

where $\gamma_{n r}^{W L}$ is the non-radiative decay term, $\Gamma_{s p}^{W L}$ is the spontaneous recombination term (that will be neglected consistently with the hypothesis of a low WL population), and $\Lambda$ is a pumping term accounting for a possible current injection into the WL, moderate enough such that Coulomb effects remain negligible. Note the diffusion coefficient $D$ which spreads out any initially localized excitation in the transverse plane and may contribute to diffusively couple QD at different locations. Again, spontaneous emission process in the WL have been disregarded when considering first order process in $N_{W L}$. For a constant spatial density of QD $N_{Q D}$, the Auger term and the capture term read:

$$
\begin{aligned}
& \left.\frac{\partial N_{W L}^{e, h}}{\partial t}\right|_{Q D-W L} ^{\text {Auger }}=-\left.N_{Q D} \frac{\partial n^{e, h}}{\partial t}\right|_{Q D-W L} ^{\text {Auger }}, \\
& \left.\frac{\partial N_{W L}^{e, h}}{\partial t}\right|_{Q D-W L}=-\left.N_{Q D} \frac{\partial n^{e, h}}{\partial t}\right|_{Q D-W L} .
\end{aligned}
$$

It is now crucial to introduce the distribution of QD heights, intrinsic of the SK growth, a phenomenon known to introduce an inhomogeneous broadening of the spectral linewidth of the dot ensemble. The contribution of each class of dots is weighed by a statistical factor,

$$
G_{\Delta_{i}}(\Delta)=\frac{1}{\Gamma / \gamma \sqrt{\pi}} \exp -\left(\Delta_{i}-\frac{\gamma}{\Gamma} \Delta\right)^{2}
$$

as determined by the detuning from the center $\omega_{i}$ of the inhomogeneously broadened line as in [22]. $\Delta_{i}=\left(\omega_{i}-\omega_{0}\right) / \Gamma$ denotes the field detuning from the QD population line-center and $\Gamma$ is the inhomogeneous QD linewidth.

The WL equation (10) is only modified through the QD-WL interaction terms $(11,12)$. Since Auger or capture processes cannot involve two different dots, the carriers captured by all the dots (which equals the total population lost by the WL) is then just the capture rate for one spectral class of dots summed over the whole distribution :

$$
\left.\frac{\partial N_{W L}^{e, h}}{\partial t}\right|_{Q D-W L} ^{\text {Auger }}=-N_{Q D} \gamma_{p} \times \int\left(\mp B_{e h} N_{W L}^{e, h} n^{h, e}\left[\Pi-n^{e, h}\right] \pm \mathrm{sym} .\right) \times G_{\Delta_{i}}(\Delta) d \Delta,
$$




$$
\begin{aligned}
& \left.\frac{\partial N_{W L}^{e, h}}{\partial t}\right|_{Q D-W L}= \\
& \quad-N_{Q D} \gamma_{p} \times \int\left(-\gamma_{e s c}^{e, h} n^{e, h}+\sigma_{c a p}^{e, h} N_{W L}^{e, h}\left[\Pi-n^{e, h}\right]\right) \times G_{\Delta_{i}}(\Delta) d \Delta .
\end{aligned}
$$

If we concentrate on the role of QD-WL interactions in determining the $\alpha$-factor in a QD-based microcavity, a fundamental feature that must be taken into account is the distribution of the dot inhomogeneous sizes which changes the spectral distribution of carrier occupancy. A deeper QD will have a larger energy gap, so that when the difference in energy between the WL and the QD is larger, the carrier lasts longer in the GS, i.e., it is more difficult for it to escape into the WL [46, 47]. The rate $\gamma_{e s c}$ is just the inverse of the escape time and hence its dependence is proportional to $\exp \left[\left(E_{Q D}-E_{W L}\right) / k_{B} T\right]$. For the carrier capture $\sigma_{c a p}$ the situation is the opposite in the sense that the transition form the WL into the excited state is favored when the difference $E_{W L}-E_{Q D}$ is larger. If we account for this mechanism in the derivation of our model, it turns out that the escape and carrier rates depend on the spectral class of the carrier considered and, after some manipulations reported in [24], should be modified as:

$$
\begin{aligned}
& \gamma_{e s c}(\Delta)=\gamma_{e s c}^{\rho} \exp \left(-\frac{\Gamma}{\gamma_{p}} \beta \Delta i\right) \exp (\beta \Delta), \\
& \sigma_{c a p}(\Delta)=\sigma_{c a p}^{o} \exp \left(\frac{\Gamma}{\gamma} \beta \Delta i\right) \exp (\beta \Delta) .
\end{aligned}
$$

Here,

$$
\beta=\hbar \gamma_{p} / k_{B} T
$$

and its typical value at room temperature is around 0.01-0.02.

Finally, we introduce the same scalings reported in [23] to make the model compact, make all the interaction terms explicit and write the final form for the carrier equations as:

$$
\begin{aligned}
\frac{d n_{Q D}^{e, h}}{d t}=-\gamma_{n r}\left[n^{e, h}+\frac{\Gamma_{s p}}{2} n^{e} n^{h}+\frac{|\mathbf{E}|^{2}}{1+\Delta^{2}}\right. & {\left[n^{e}+n^{h}-\Pi\right] } \\
\pm B_{h e} N_{W L}^{h} n^{e}\left[\Pi-n^{h}\right] & \mp B_{e h} N_{W L}^{e} n^{h}\left[\Pi-n^{e}\right] \\
& \left.+\gamma_{e s c}^{e, h} n^{e, h}-\sigma_{c a p}^{e, h} N_{W L}^{e, h}\left[\Pi-n^{e, h}\right]\right],
\end{aligned}
$$




$$
\begin{array}{r}
\frac{\partial N_{W L}^{e, h}}{\partial t}=-\gamma_{n r}^{W L}\left[-\Lambda+N_{W L}^{e, h}-D \nabla_{\perp}^{2} N_{W L}^{e, h}\right. \\
\mp B_{h e} N_{W L}^{h} \int n^{e}\left[\Pi-n^{h}\right] G_{\Delta_{i}}(\Delta) d \Delta \pm B_{e h} N_{W L}^{e} \int n^{h}\left[\Pi-n^{e}\right] G_{\Delta_{i}}(\Delta) d \Delta \\
-\gamma_{e s c}^{e, h} \int n^{e, h} G_{\Delta_{i}}(\Delta) d \Delta \\
\left.-\sigma_{c a p}^{e, h} N_{W L}^{e, h} \int\left[\Pi-n^{e, h}\right] G_{\Delta_{i}}(\Delta) d \Delta\right] .
\end{array}
$$

For our purposes, the most important scaling going from Eq. (9) to Eq. (19) is the one of the field via the saturation field strength

$$
E_{s}=\sqrt{\frac{\hbar^{2} \gamma_{p} \gamma_{n r}}{2 \mu^{2}}}
$$

The saturation intensity is given by

$$
I_{s}=\frac{c n_{b} \varepsilon_{0} \hbar^{2} \gamma_{p} \gamma_{n r}}{\mu^{2}}
$$

$n_{b}$ denoting the background refractive index. The (normalized) susceptibility $\chi_{I}$ of the inhomogeneously broadened QD population then stems from the summation of the responses of individual QD weighed by their Gaussian statistical contribution,

$$
\chi_{I}\left(\Delta_{i}, n_{e}, n_{h}\right)=\int \frac{1-i \Delta}{1+\Delta^{2}}\left(n^{e}+n^{h}-\Pi\right) G_{\Delta_{i}}(\Delta) d \Delta .
$$

In order to make a connection to experiments, the scaled units need to be related to real ones. The unscaled susceptibility is:

$$
\chi\left(\Delta_{i}, n_{e}, n_{h}\right)=\left(\frac{\mu^{2} N_{D}}{\hbar \varepsilon_{0} \gamma_{p}}\right) \chi_{I}\left(\Delta_{i}, n_{e}, n_{h}\right) .
$$

Here, $N_{D}$ defines an effective volume density. In vertical-cavity devices (VCSEL),

$$
N_{D}=\frac{N_{Q D} N_{l}}{L_{A}}
$$

whereas in edge-emitting devices or lasers (EEL)

$$
N_{D}=\frac{N_{Q D} N_{l}}{d},
$$

where $N_{l}$ is the number of QD layers, $d$ is the total thickness of the active zone (EEL) and $L_{A}$ the total length of the active zone (VCSEL). From this, the absorption coefficient for the intensity can be obtained as 


$$
\alpha\left(\Delta_{i}, n_{e}, n_{h}\right)=\frac{\omega_{0}}{n_{b} c} \operatorname{Im} \chi\left(\Delta_{i}, n_{e}, n_{h}\right) .
$$

Analogously, the refractive index can be determined from the real part of the scaled susceptibility as:

$$
n=\frac{1}{2 n_{b}} \chi=\Delta n_{0} \operatorname{Re} \chi_{I}
$$

where $\Delta n_{0}$ is equal to

$$
\Delta n_{0}=\frac{\mu^{2}}{2 n_{b} \hbar \varepsilon_{0} \gamma_{p}} N_{D} .
$$

Note that these are the material coefficients, to obtain the modal coefficients for devices in which there is only a partial overlap between the active region and the field distribution (e.g. in EEL) one needs to multiply with a confinement factor $\Gamma_{\text {trans }}$, which is given by an overlap integral over the fast direction between the fundamental waveguide mode intensity distribution and the thin active layer.

\subsubsection{Cavity equation}

The field equation is derived here in the case of a broad area resonator, by following the same procedure as in $[48,49]$. In the mean-field limit and introducing the appropriate scalings as in [23], we can write the equation for the intracavity field as:

$$
\frac{\partial E}{\partial t}=-\left[(1+i \theta) E-E_{I}-i \nabla_{\perp}^{2} E-2 C \chi\left(\Delta_{i}, n_{e}, n_{h}\right) E\right] .
$$

The time here has been scaled to the field decay rate $\kappa=c T / 2 n_{b} L$ in the cavity. We also have introduced $\theta=\left(\omega_{c}-\omega_{0}\right) / \kappa$, the scaled cavity-field detuning and the cooperativity parameter

$$
2 C=\frac{\mu^{2} \omega_{0} N_{Q D}}{\varepsilon_{0} \hbar \gamma_{p} n_{b} c T} .
$$

$E_{I}$ is an injected field while the transverse Laplacian $\nabla_{\perp}^{2}$ accounts for the diffraction inside the cavity. The spatial transverse coordinates are rescaled to the diffraction coefficient $a=c / 2 n_{b} k_{o} \kappa . L$ is the cavity length and $T$ the mirror transmission.

Equations (19) for the electrons and holes and (20) for the WL with Eq. (30) for the field are the self-consistent set for a general description of a broad-area QD microresonator. (Due to the scaling of time with $\kappa, \gamma_{n r}$ and $\gamma_{n r}^{W L}$ in Eqs. (19), (20) need to be scaled also to $\gamma_{n r} / \kappa$ and $\gamma_{n r}^{W L} / \kappa$.)

\subsubsection{Propagation equations}

The paraxial wave equation describing single-pass propagation of a light field $E(x, x, z)$ in a medium with a susceptibility $\chi$ is 


$$
\partial_{z} E=i \frac{c}{2 n_{b} \omega_{0}} \nabla_{\perp}^{2} E+i \frac{\omega_{0}}{2 n_{b} c} \Gamma_{\text {trans }} \chi E
$$

The transmitted field after a thin layer of matter with thickness $\delta z$ is (thin enough such that diffraction can be neglected)

$$
E(x, y, z+\delta z)=\exp \left[i \frac{\omega_{0}}{2 n_{b} c} \chi(x, y, z) \delta z\right] E(x, y, z)
$$

In most investigations presented below, we will assume that this description is fine for the whole sample, i.e. we set $\delta z=L_{A}$ neglecting pump depletion (or amplification) and diffraction within the medium.

\subsection{Numerical results: Single-pass propagation}

\subsubsection{Parameters and numerical scheme}

Calculations can be performed for the (two-dimensional) case of a surface-emitting geometry or for a (quasi-one-dimensional) edge-emitter. We concentrate on the latter because of the smallness of the optical density in a surface-emitter. Then, Eqs. (19), (20) are solved numerically for a cw Gaussian input beam $E(x)=$ $E_{0} \times \exp \left(-x^{2} / w_{x}^{2}\right)$ on a numerical grid with 64 space points and a beam waist $w_{x}$ of 15 points or $15 \mu \mathrm{m}$. We resolve spectrally 61 size classes. About 8000 iterations are needed until the solutions to the carrier equations relax to the stationary state. Due to the thinness of the active zone in the fast direction $(y), E(x)$ can be taken as the peak value of the field profile of the fundamental mode of the waveguide in the fast direction (with radius $w_{y}$ ) with the form $E(x, y)=E(x) \times \exp \left(-y^{2} / w_{y}^{2}\right)$. Note that we do not consider any built-in waveguide in the $x$-direction. The resulting spatial distributions $n^{e}(x)$ and $n^{h}(x)$ are then used to calculate the spatial distribution of the susceptibility by Eqs. (23) and (24).

In the experiment, one is not measuring the absorption coefficient (27) directly but transmission. Typically, the latter will be integrated over the beam in addition:

$$
T=\int \exp \left[-\Gamma_{\text {trans }} \alpha(x) L_{a}\right]\left(\frac{2}{\pi}\right)^{1 / 2} \frac{1}{w_{x}} \exp \left(\frac{-2 x^{2}}{w_{x}^{2}}\right) d x
$$

where

$$
\Gamma_{\text {trans }}=\frac{\int_{-d / 2}^{d / 2}|E(y)| d y}{\int|E(y)| d y}
$$

is the confinement factor for an edge-emitting structure.

We are choosing parameters typical for InAs QD emitting in the $1.3 \mu \mathrm{m}$ region at room temperature $[50,51,15,9,16]$. We assume the following set of parameters in normalized units: $B_{e h, h e}=200, \sigma_{c a p}=500, \gamma_{e s c}^{e}=0.01, \gamma_{e s c}^{h}=100, \gamma_{n r}^{W L}=0.15$ 
and a time unit of $11.7 \mathrm{ps}$. This translates to a rate of (1/160 fs) for the capture and Auger processes coupling the WL to the QD in agreement with measurements for the refilling of the QD ground state from WL and excited states [16, 9].

Other parameters are $[51,15,52]: N_{Q D}=5 \times 10^{10} \mathrm{~cm}^{-2}$ QD dot sheet density, $N_{l}=10, d=10 \times 3.9 \times 10^{-8} \mathrm{~m}, \omega_{0}=1.45 \times 10^{15} \mathrm{~s}^{-1}, \lambda=1.3 \mu \mathrm{m}, \beta=0.02$, $L_{a}=1 \mathrm{~mm}, \gamma_{p}=7.1 \times 10^{12} \mathrm{~s}^{-1}, \Gamma / \gamma_{p}=4$, corresponding to an inhomogeneous broadening of about $40 \mathrm{~nm}$. A dipole matrix element of $\mu=1.23 \times 10^{-28} \mathrm{Cm}$ corresponding to a radiative lifetime of $1 / \Gamma_{1}=0.5 \mathrm{~ns}$ results then in a small-signal modal absorption coefficient in line center (Eq. (27) for $n_{e}=n_{h}=0, \Gamma_{\text {trans }}=0.094$ ) of $\alpha_{0}=26.7 / \mathrm{cm}$. Since reported small-signal modal gain values for these structure are between about $19-24 / \mathrm{cm}$ in the $1250-1290 \mathrm{~nm}$ range [51,9] with small-signal absorption being about 30-100\% larger than the small-signal gain [9], this is a conservative estimate. Note that in the remainder of Sec. 2 and in Sec. $3 \alpha, \alpha_{0}, n, C$ denote modal absorption coefficients, refractive indices and cooperativity parameters, whereas in Sec. 4 they denote the material parameters.

\subsubsection{Results: Saturation of absorption and gain}

Fig. 1 displays the gain, respectively absorption, coefficient obtained from Eq. (34) in dependence of the input intensity for different detunings. For all curves, it starts at the small-signal value and then drops to the vacuum value of zero due to the generation of carriers and the resulting bleaching. Obviously, the small-signal absorption/gain is highest at $\Delta_{i}=0$ and decreases for increasing modulus of detuning according to Eq. (27). The intensity where saturation becomes apparent seems to increase with increasing modulus of detuning.

Since the ratio of $\Gamma / \gamma_{p}=4$ refers to a Voigt-profile situation where neither homogeneous nor inhomogeneous broadening are clearly dominating, we fit the dependence of the gain coefficient on intensity with different models that describe saturable absorption in the case of two-level systems with inhomogeneous,

$$
\alpha=\alpha_{0} / \sqrt{\left(1+E^{2} / E_{s}^{2}\right)}
$$

and homogeneous,

$$
\alpha=\alpha_{0} /\left(1+E^{2} / E_{s}^{2}\right)
$$

broadening [53]. The latter proves to fit best the simulation. We show in Fig. 2, the modulus squared of the saturation field strength vs $\Delta_{i}$ as extrapolated from the above formula. The saturation intensity is minimal at $\Delta_{i}=0$ and is slightly different, about a factor of two, for the gain $\left(E^{2}=3.6\right)$ and the absorption case $\left(E^{2}=1.8\right)$. It strongly increases in both cases for increasing modulus of the detuning, whereas it should be constant in the strongly inhomogeneous limit [53]. As indicated above, a ratio of $\Gamma / \gamma_{p}$ corresponds neither to strongly inhomogeneous broadening nor to pure homogenous one. Additional coupling is provided by the fact that different size classes of QD are coupled via the escape and recapture processes via the WL 


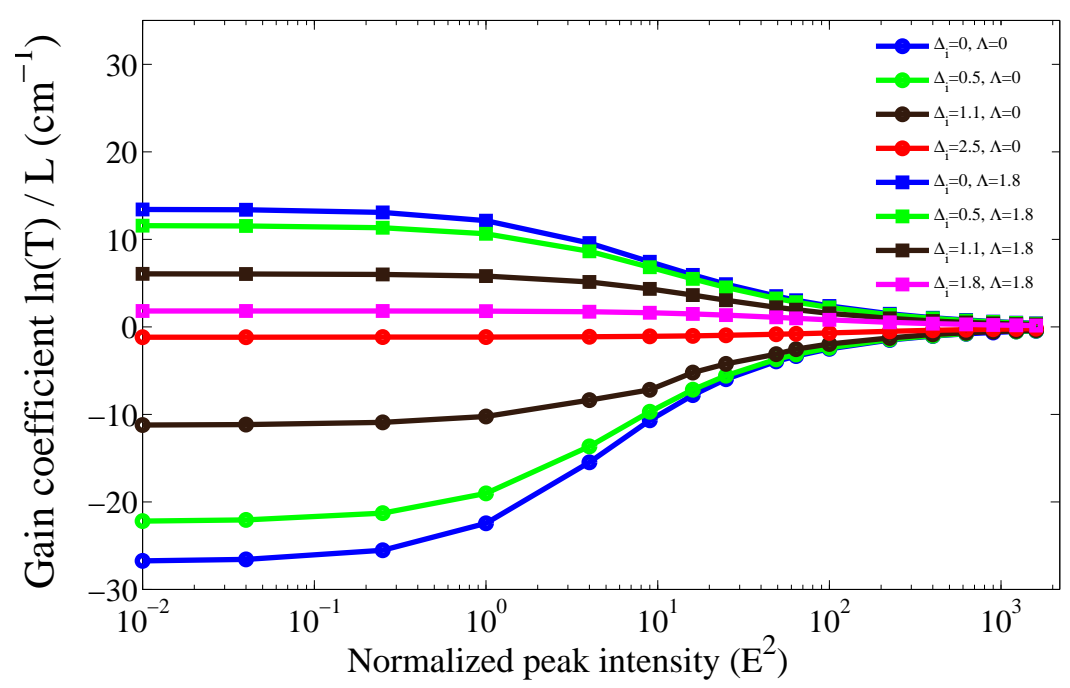

Fig. 1 (Color online) Modal gain coefficient as a function of the normalized intensity in the center of the Gaussian input beam for different values of the detuning in the absorption (circles) and gain regimes (squares). [Adapted from [25]]

and lead to a stronger homogeneous broadening at room temperature than at lower temperatures [54]. Nevertheless, at least in quantum dash samples there is evidence for a strong coherent hole (the equivalent to the so-called Lamb-dip in Dopplerbroadened atomic ensembles) at room temperature [55] indicating at least partial inhomogeneous broadening. This coherent hole is observed in other simulations also. Hence it seems to make sense that the saturation behavior shares features known from homogeneous and inhomogeneously broadened systems.

For an experimental situation with $w_{x}=15 \mu \mathrm{m}$ and $w_{y}=0.5 \mu \mathrm{m}, E^{2}=1$ corresponds to a power of $7.1 \mathrm{~mW}$. Hence the minimum value of the saturation power is $13 \mathrm{~mW}$ in the absorption case and $26 \mathrm{~mW}$ in the gain case. If instead of $\gamma=0.15$ (or $78 \mathrm{ps}$ lifetime), the purely radiative lifetime is considered, the corresponding values are about a factor of 10 lower and easily accessible experimentally.

As indicated, the simulations presented neglect pump depletion within the medium. We have preliminary results using a split-step beam propagation method [56]. The term 'split-step' implies that the simultaneous action of diffraction and nonlinear refraction (determined by $\chi$ ) in Eq. (32) is replaced by a step-wise scheme of alternating diffraction and refraction steps. This works by splitting the medium in $m$ layers with a thickness $\delta z=L_{A} / m$. The diffraction part is solved in Fourier (transverse wave number) space, the refraction is solved in real space via Eq. (33). In each layer the carrier equations need to be solved providing a significant computational load. We find that for our case, $m=16$ provides enough resolution (for $L_{A}=1 \mathrm{~mm}$ ) so that the results do not depend on discretization. The principal shape of the saturation curves is not strongly affected by taking into account pump depletion but 


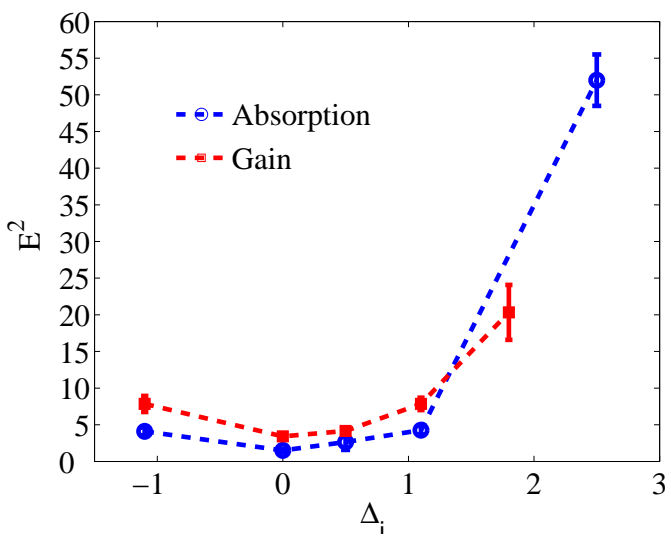

Fig. 2 (Color online) Modulus squared of saturation field strength (proportional to saturation intensity) obtained from a fit of the curves in Fig. 1 to Eq. (36) as a function of detuning. Squares represent the gain case (red online), circles the absorptive case (blue online). The lines are only a guide for the eye. [Adapted from [25]]

the saturation intensity for the absorption increase by a factor of about 1.6 and the saturation intensity for the gain decreases by a factor of about 0.7 (for $\Delta_{i}=0$ ). As a result, the gain saturates now slightly easier than the absorption. This is easily understandable because the pump depletion due to absorption will hinder saturation in the subsequent layers. On the other hand, the amplification due to gain will help to saturate the gain in the subsequent layers. Similar considerations will be important in Sec. 3 to interpret multi-pass effects.

\subsubsection{Results: Self-lensing}

One effective method to assess the strength of a $\chi_{3}$ - or saturable refractive index nonlinearity is to look for self-lensing, e.g. in a so-called z-scan geometry [57]. Since the input beam is spatially varying, also the refractive index is. Around the beam center, the variation is necessarily parabolic. According to [58] the radius of curvature acquired by a wave propagating a distance $L_{a}$ in a medium is given by

$$
\frac{1}{R(r)}=\frac{1}{r} \frac{\partial}{\partial r} n(r) L_{a}
$$

From that we can identify an effective focal power:

$$
\begin{aligned}
\frac{1}{f}(x) & =-\frac{1}{x} \frac{\partial}{\partial x} n(x) L_{a} \\
& =-\Delta n_{0} L_{a}\left(\frac{1}{x} \frac{\partial}{\partial x} \operatorname{Re} \chi_{I}(x)\right)
\end{aligned}
$$


If the refractive index distribution would be a pure parabola, the focal power would be constant over the whole beam, thus implying an aberration-free equivalent lens. In reality, this is obviously not the case because the pumping Gaussian has an inflection point. Nevertheless the parabola is often a good approximation in beam center where most of the beam energy is. This was studied in detail in atomic vapors [59] and we will discuss it for the QD below. In any case, the curvature will give a quantitative indicator for the strength of beam shaping even if the lens is not perfect. The focusing can be experimentally detected by a change of the beam width in far field [60] or at some distance after the medium [59] (similar as in z-scan techniques [57]). In this first treatment, we will confine to a thin lens to demonstrate the principles. For a quantitative description of a real experiment it might be necessary to include absorption and nonlinear beam reshaping during propagation.

Fig. 3 shows the spatial profile of the real part of the susceptibility imposed by the Gaussian pumping profile for low, intermediate and high peak intensity. For low intensity it follows roughly the Gaussian intensity distribution of the input beam ('Kerr-limit') whereas at high intensities there is a broad plateau in beam center because the beam has sufficient intensity to saturate the sample even in the beam wings. At beam center, the variation is parabolic leading to lensing. Fig. 4 shows

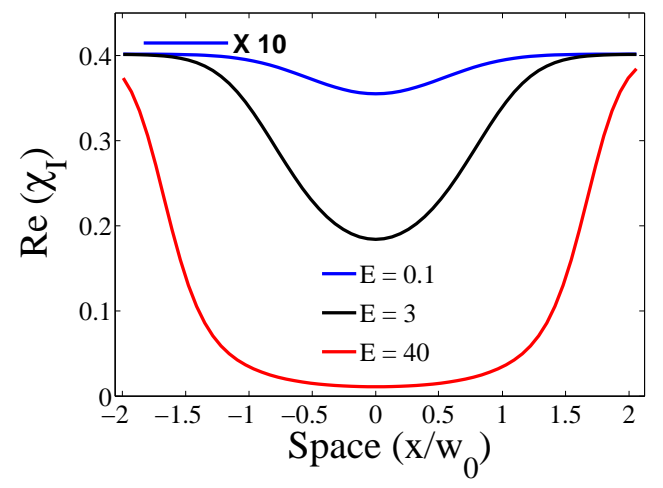

Fig. 3 (Color online) Spatial profile of the real part of the susceptibility for, from top to bottom line, low (blue line, amplitude enhanced by a factor of 10), intermediate (black line) and high excitation (red line). $\Lambda=0, \Delta_{i}=1.1$. The total excursion is $\Delta n=1.6 \times 10^{-4}$. [From [25]]

how the lens power change as function of input intensity for different detunings in the absorption case (a) as well as in the gain case (b). Apart from the $\Delta_{i} \approx 0$-case (discussed separately below), the focal power increases from zero with increasing intensity, reaches a peak at an intermediate intensity and decreases again if the intensity is increased further. The sign of the lensing depends on the sign of detuning and whether the sample is absorbing or providing gain, as expected. The maximum lens effect occurs at $\Delta_{i}=1.1$ and $E^{2}=9(P=64 \mathrm{~mW})$. The focal power is maximum at an intermediate input power a few times higher than the saturation power. The 
intensity needed to obtain maximum lens power increases for increasing modulus of detuning. This is probably due to the fact that the saturation intensity increases with detuning and the maximum effect is found for the same saturation condition.
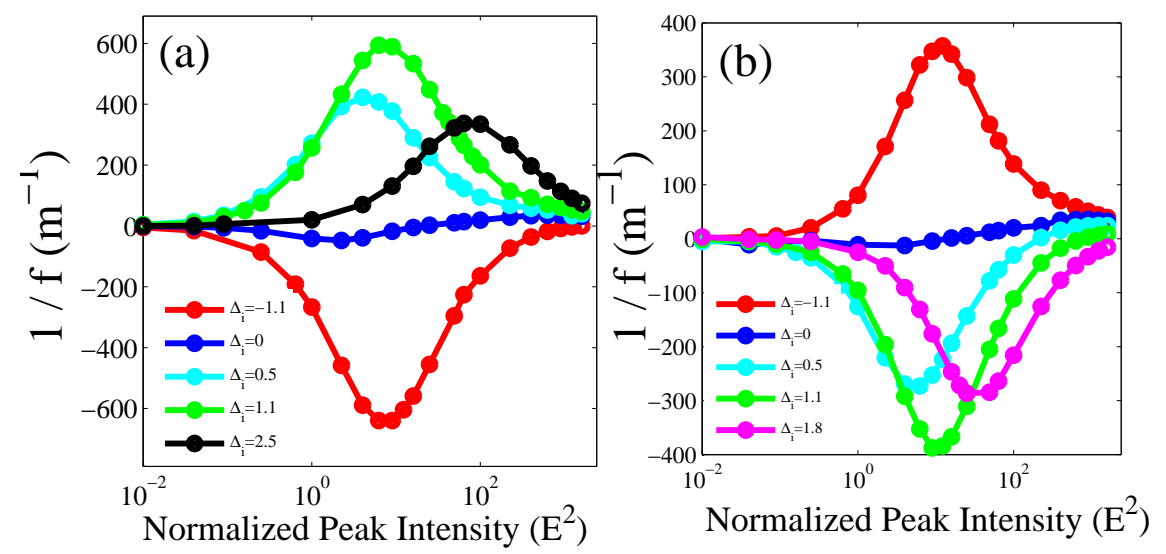

Fig. 4 (Color online) a) Focal power as function of normalized peak intensity for different detunings in the absorption case (a) and the gain case (b). $(\Lambda=1.8$ was chosen because it reproduced the experimental finding that maximal gain is about half the absorption coefficient.) [From [25]]

The fact that the maximum focal power is obtained at intermediate input intensity can be explained by looking at Fig. 3. For low intensity the curvature follows the curvature of the input profile ('Kerr-limit', $\Delta n(x) \sim|E|^{2}(x)$ ), but the total effect is low because the excursion from the background refractive index is small (note that the curve is blown up by a factor of 10). For high intensity, the excursion is large $\left(\operatorname{Re}\left(\chi_{I}\right)\right.$ becomes nearly zero) but the total focal power is again low because the curvature is strongly reduced. This is due to the fact that saturation is effective over a large area at high intensities. The case of intermediate intensity is in between: On the one hand the excursion is of reasonable size, about half the maximal effect, on the other hand the curvature is still quite close to the one of the input beam. Both is characteristic for intensity levels around the saturation intensity, i.e. for the onset of saturation, and hence the total effect is maximal. Similar characteristics were found for atomic vapors [59]. Here, in the homogeneously broadened case, it can be demonstrated analytically that maximum focal power is found at the saturation power [59].

The lensing effect is minimal at $\Delta_{i}=0$. Indeed, in a purely two-level system no effect at all is expected for $\Delta_{i}=0$ because the contributions of blue and red detuned size classes cancel. It is the thermally induced coupling to the WL (described by the parameter $\beta$ ) which breaks that symmetry (see Sec. 2.3.1). 
In the peak, the predicted lensing effect is actually quite substantial, $\left|f_{\text {min }}\right| \approx$ $1.7 \mathrm{~mm}$, in a sense, because the focal length reaches the length of the medium (assumed to be $L_{a}=1 \mathrm{~mm}$ ), i.e. the point where the approximation by a thin lens becomes questionable. These values were calculated assuming an input beam radius of $w_{x}=15 \mu \mathrm{m}$ chosen because it would be conveniently to work with experimentally and being somewhat larger than typical fundamental mode sizes in edge-emitting lasers, i.e. in a range where filamentation phenomena might occur. The size of cavity solitons is also in that range (about $10 \mu \mathrm{m}[30,61]$ ).

Nevertheless, it turns out that an experimental confirmation is not straightforward: The modification of the input beam by the lensing of the sample can be detected by either measuring the on-axis amplitude (being proportional to the square of the new beam waist of the transmitted beam, $w_{x}^{\prime 2}$ ) or the beam width in far field $\left(\sim 1 / w_{x}^{\prime 2}\right)$ [60] or, more sensitively, by measuring the beam size either directly or via the transmission through a pinhole at some suitable chosen distance after the medium as it is done in usual z-scan techniques [57]. Replacing the medium by a thin lens of focal length $f$, the size of the new beam waist $w_{x}^{\prime}$ can be calculated by ABCD-matrix theory as

$$
w_{x}^{\prime}=w_{x} \frac{1}{1+\frac{\pi w_{x}^{4}}{\lambda^{2} f^{2}}} .
$$

For an input beam waist of $w_{x}=15 \mu \mathrm{m}$ and a thin lens with $f \approx 1.7 \mathrm{~mm}$, the new beam waist is $w_{x}=14.3 \mu \mathrm{m}$ at a distance of $0.16 \mathrm{~mm}$. This rather small change in beam is quite difficult to detect. Eq. (41) says that the effect becomes more pronounced if the initial beam radius is increased (at constant $f$ ), being substantial if the Rayleigh length of the input beam $z_{r}=\pi w_{x}^{2} / \lambda$ is of the order of $f$. In reality, however, the focal length scales like $f \sim w_{x}^{2}$, since, as discussed for Fig. 3, the curvature of the susceptibility profile follows the curvature of the input beam in first approximation for not too strong saturation (see [59] for an analytical treatment). Hence, actually the strength of the detected signal can't be influenced by choice of the input beam size.

However, due to the approximately quadratic dependence of the new beam waist on ratio of $w_{x}^{2} / f$, the situation rapidly improves with increasing focal power. For example, a change of size by $20 \%$, which should be experimentally detectable, is reached already for a focal power of about $1000 / \mathrm{m}$, i.e. only about two times the maximum value reported in Fig. 4.

Since it appears that the numbers are somewhat at the edge, it is useful to discuss the influence of other uncertainties, e.g. the exact nature of the relaxation processes between the QD and WL states. Results reported in [25] indicate that the changes are within the $20 \%$ range for the dominating part (real or imaginary) of the susceptibility for reasonable adjustments of parameters. The carrier lifetime in the QD was assumed be $78 \mathrm{ps}$, much smaller than the radiative lifetime of $500 \mathrm{ps}$. This was done on the one hand to be on the conservative side with respect to the possible influence of defect induced recombination and on the other hand to reduce the computational load, which is rather high due to the separation of time scales between scattering processes between WL and QS and carrier lifetime in QD and due to the fact that 
the carrier density needs to be spectrally and spatially resolved in our case. We did some test runs using a lifetime of $0.5 \mathrm{~ns}$ which yield an increase of $10 \%$ in saturation and negligible effect in lensing. Note that the influence of the lifetime on the scaling of the saturation power can be treated exactly without additional calculations (as discussed above) due the way the equations are scaled.

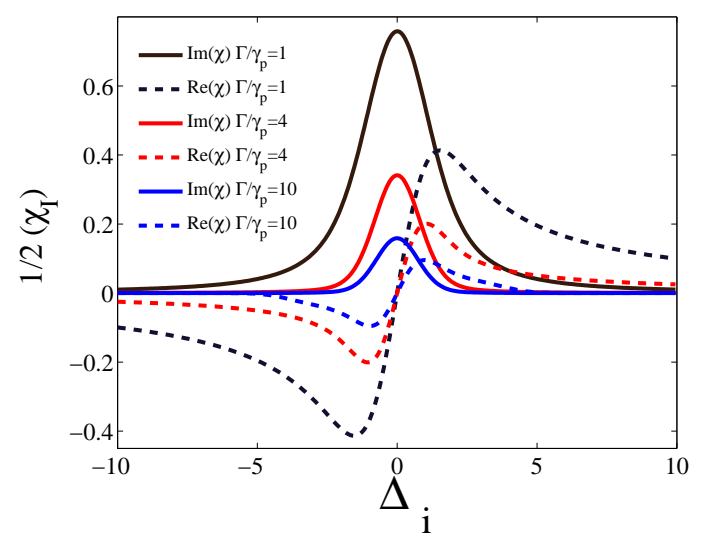

Fig. 5 (Color online) Real and Imaginary part of the linear susceptibility as function of detuning for different ratios between inhomogeneous and homogeneous broadening. [From [25]]

Finally, Fig. 5 shows how real and imaginary part of the linear susceptibility change as function of detuning for different ratios between homogeneous and inhomogeneous broadening. Since the linear susceptibility defines the maximum value of the nonlinear index change, this provides a good guidance on the maximum effect to be expected. Choosing a ratio of $\Gamma / \gamma_{p}=10$ instead of 4 decreases the maximum of the real part of the susceptibility by a factor of 2.1 . For a ratio of $\Gamma / \gamma_{p}=1$, it is a factor of 2 higher. Hence, at constant $\gamma_{p}$, one can expect to benefit from improved growth with a reduced inhomogeneous broadening. Note that increasing $\gamma_{p}$ at constant $\Gamma$ is not beneficial because the increase of the scaled susceptibility is sublinear (cf. Fig. 5) and is overcompensated by the dependence of the proportionality factor between scaled and unscaled susceptibility on $\gamma_{p}$, see Eq. (24). We conclude that though uncertainties in the relaxation constants will influence the measurements quantitatively, our overall conclusion that the lensing is at the edge of being detectable is not changed. One reason for the somewhat low nonlinear phase shift is the inhomogeneous broadening. The peak phase shift from Fig. 5 for $\Gamma / \gamma_{p}$ is only $40 \%$ of what a homogenously broadened transition with the same total QD density would give.

At this point it is worthwhile to note that a possible susceptibility contribution of the WL carrier population or ES population should be introduced here. Taking for example the curve with $\Gamma / \gamma_{p}=4$ considered relevant here, the refractive index 
contribution of an ES 100-120 nm away from the GS (roughly the situation in InAs QD) will partially overlap and counteract the effect of the GS transition: With an inhomogenous broadening of $\Gamma \approx 40 \mathrm{~m}$ the distance between the ES and the GS on the $\Delta_{I}$-axis in Fig. 5 is 3 . (The contribution is smaller for the imaginary part of the susceptibility (our main focus in the experiment later) because that tails off fast than the real part with detuning.) In particular that implies that there might be a nonzero $\alpha$-factor around the gain peak of the GS due to the off-resonant contributions from WL and ES. Though there are reports of fairly low $\alpha$-factors [35, 36, 37] on the one hand, there is also significant evidence of contributions from the other states, discrete or continuous (WL or barrier based), whose contribution to the refractive index in particular could be significant for high injection values (and thus large carrier densities in the ES or WL) $[38,39,40]$. Modeling more complex contributions has been dealt with by means of properly balanced rate-equations models for the carriers and/or by inclusion of a contribution from the continuum and discrete states in WL and QD either in a semi-phenomenological or more first-principle way $[39,62,63,64,65]$.

\subsection{Numerical results: Cavity dynamics}

\subsubsection{Nonlinear refractive index and $\alpha$-factor}

As just discussed, the $\alpha$-factor is an important, but still controversial factor in QDbased photonic devices. It depends very much on operating conditions and also on measurement method (see, e.g., the discussion in [39]). Commonly used techniques to measure the $\alpha$-factor (e.g. [39]) are based on the FM/AM response of the laser output or the amplified spontaneous emission spectrum to a small modulation or variation of the injection current using the relation

$$
\alpha_{H}=\frac{\frac{d \operatorname{Re} \chi}{d N}}{\frac{d \operatorname{Im} \chi}{d N}},
$$

where the change in carrier density (being it in the QD or the WL) is introduced via the variation of current around some working point. In another method one analyzes the output of a laser with injection [66]. Since this latter method can be easily described by our formulation, Eq. (30), we use it here to point out one aspect of the carrier dynamics leading to an asymmetric gain spectrum and hence a nonzero $\alpha_{H}$ at gain peak in addition to the off-resonant effects of the WL and ES states. This is the different thermal occupation of the size dispersed QD GS states described by the factor $\beta$ introduced in Eq. (18).

We considered a pumped device with currents spanning from absorption to gain and evaluated the $\alpha$-factor defined as in [24]. For vanishing $\beta$ (meaning the neglect of the thermal distributions of the capture and escape rates) a symmetric, two-level like profile of the gain spectrum is maintained as shown in Fig. 6. The inhomogenous 
broadening does not influence the symmetry but decreases only the peak effect (as discussed above).
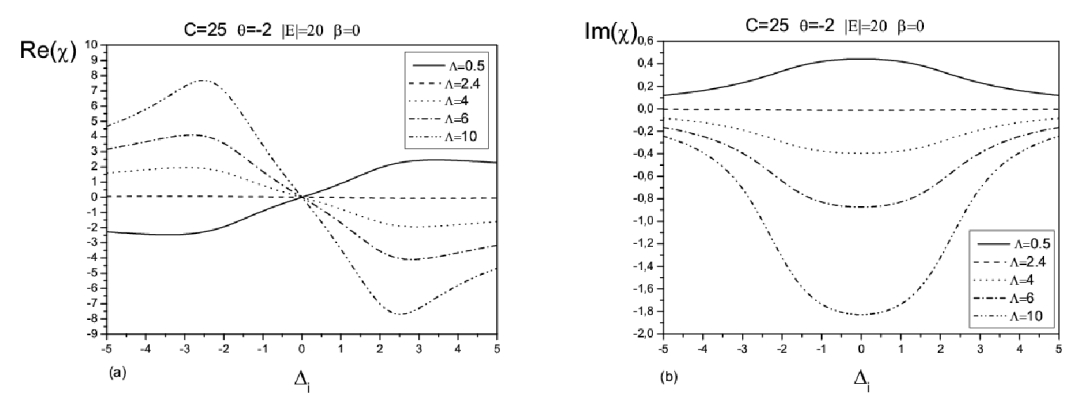

Fig. 6 Plot of the real and imaginary parts of the susceptibility spectrum versus the detuning from the QD population line center for $\beta=0$ and for five values of the pump $\Lambda$ from absorption to gain. Other parameters for these simulations are $\sigma_{c a p}^{e, h}=500, \gamma_{e s c}^{h}=100, \gamma_{e s c}^{e}=0.01, B_{h e, e h}=500$, $\gamma_{p}=15, \Gamma=60, \Gamma_{s p}=2.5, \gamma_{n r}=\gamma_{n r}^{W L}=0.15, C=25, \Theta=-2,|E|=20$. [From [24]]

Fig. 7 shows the situation for $\beta=0.02$. When $\beta>0$, the gain spectrum shows an asymmetry steadily growing with $\beta$ and with carrier injection $\Lambda$ (Fig. 7b) thus implying a nonvanishing $\alpha$-factor. The real part of the susceptibility (Fig. 7a) is less affected, the point where all dispersion curves approximately intersect and hence where $\alpha_{H} \approx 0$ moves to slightly positive detuning (here $\Delta_{i} \approx 0.015$ ).
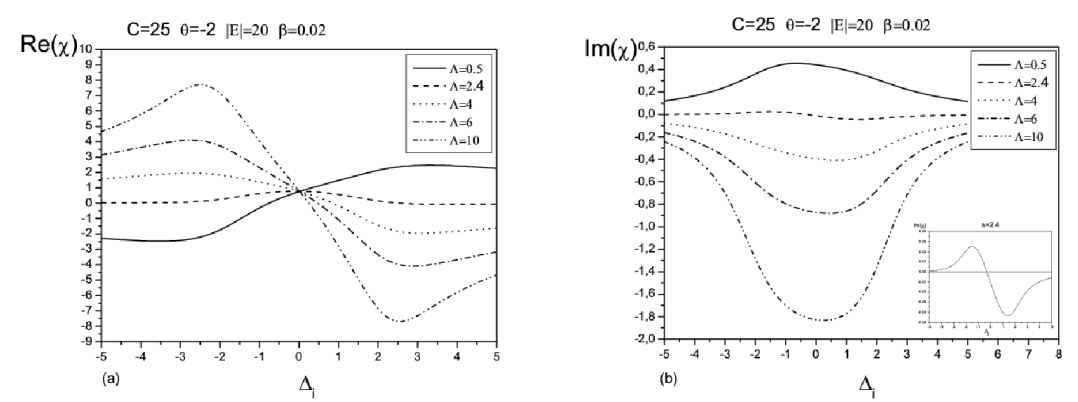

Fig. 7 Plot of the real and imaginary parts of the susceptibility spectrum versus the detuning from the QD population line center for $\beta=0.02$ and five values of the pump $\Lambda$ from absorption to gain. Other parameters as in Fig. 6. The inset is a blow-up of the curve for $\Lambda=2.4$. [From [24]]

The $\alpha$-factor can be evaluated by using the definition at the beginning of this section, i.e. varying $\Lambda$ by a small amount around a working point and calculating 
the resulting differentials numerically. For a bias of $\Lambda=2.36$ (pump slightly below transparency) and $\beta=0.01$, we plot its value in Fig. 8 .

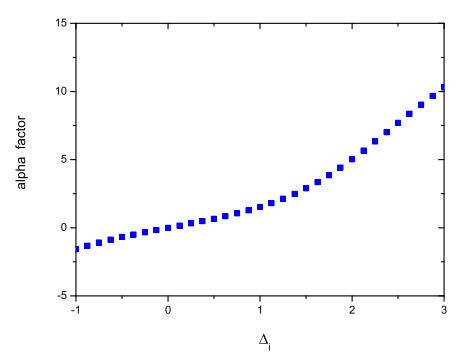

Fig. $8 \alpha$-factor versus the detuning from the QD population line center for $\beta=0.01$ and $\Lambda=2.36$. Other parameters as in Fig. 6 except $C=20, \Theta=-3,|E|=30$.

By inspecting it, we see that for fixed current pump (below threshold) and input field values, the system exhibits a negative $\alpha$-factor for higher energy (smaller wavelength) spectral values, consistently e.g. with [62], which increases to positive values, again, with a behavior qualitatively not dissimilar from the ones reported e.g. in Fig. 6 of [67], and experimentally in [66]. The dispersion curve is strongly asymmetric, though the zero point is actually not much shifted for this relatively low values of $\beta$.

\subsubsection{Nonlinear phase shift and Fabry-Perot fringes}

We discussed in Sec. 2.2.3 self-lensing as a possible method to assess the nonlinear, intensity-dependent, phase shift and found that it is close to the detection limit. Interferometric methods are an attractive alternative in which a probing beam after having passed the QD sample might be interfered with a reference beam. Alternatively, one might measure the shift of cavity resonances with intensity. Note that contrary to the way the $\alpha$-factor is normally measured, i.e. by changing the carrier density via changing the injection current (see previous section), we are interested here in the index shift generated by a coherent light beam via the carriers it generates (or takes out via stimulated emission).

In a Fabry-Perot cavity a refractive index change of $\delta n$ causes a shift of the wavelength resonance by

$$
\delta \lambda=\lambda_{0} \frac{\delta n}{n_{b}},
$$

where $\lambda_{0}$ is the resonance wavelength belonging to the background index $n_{b}$. In order that a shift is detectable, it should be about half of a free spectral range (FSR) of the cavity given by $\Delta \lambda_{F S R}=\lambda_{0}^{2} /\left(2 n_{b} L\right)$. From this the detectable refractive index should be 


$$
\delta n=\frac{\lambda_{0}}{4 L} .
$$

For the simulation parameters used in Sec. 2.2.1, the maximum index shift is $1.6 \times$ $10^{-4}$ at $1280 \mathrm{~nm}$. Hence, the nonlinear shift should be detectable in cavities of length of 2-4 $\mathrm{mm}$. Note that this is only a rough estimation. On the one hand a shift of the fringes of less than half a FSR is detectable, on the other hand, the numbers given assume optimal tuning for the maximal index effect (Fig. 5) and complete saturation.

\subsubsection{Optical Bistability}

The set of equations proposed in Sec. 2.1 can be directly exploited to investigate the stationary emission states of a microresonator with coherent injection. In particular, an interest resides in the regimes where the stationary curve has a bistable character. This issue is interesting 'per se' for all-optical processing applications, but also because bistability is the fingerprint of highly nonlinear regimes and has also been investigated in relation to the search for modulational instabilities leading to pattern formation and cavity solitons (see, e.g., [27, 28, 29] for reviews).
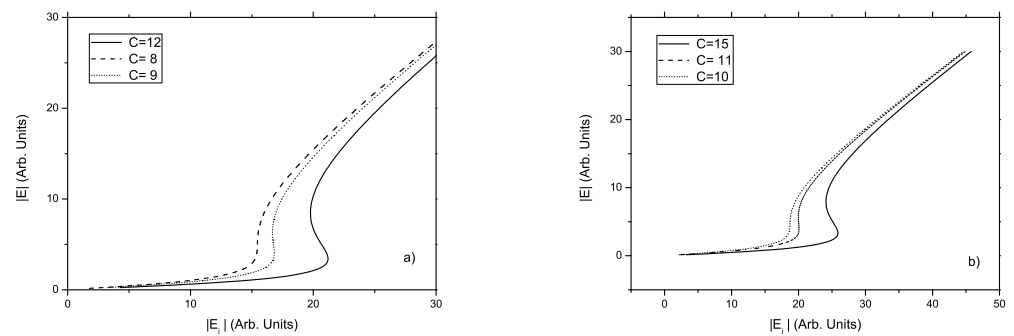

Fig. 9 Bistable and monostable steady state curves for the intracavity vs injected field amplitudes: a) resonant case $\theta=\Delta_{i}=0$ with $C=12,9,8$ (respectively, full, dotted and dashed lines) representatives of bistable, threshold and monostable regimes; b) dispersive case $\theta=-1, \Delta=0$ with $C=15,11,10$ (respectively, full, dashed and dotted lines); representatives of bistable, threshold and monostable regimes. Other parameters as specified in the text.

In this research, the mainstream of the experimental investigations has been performed in absence of carrier pumping and with an injection resonant with the QD centerline and the cavity reference frequency (purely absorptive regime); in Fig. 9a we show the steady state field curves for the case $\theta=0, \Delta_{i}=0$ and we evidence the existence of a threshold in the $C$ parameter, approximatively equal to 9 , below which the curve is monostable. Other parameters in the simulations are the same as in Subsec. 2.3.1.

In the dispersive case, when the injected field is detuned from the cavity resonance, the bistability conditions are somewhat more demanding due to the dimin- 
ished fraction of the spectral population distribution resonant with the external drive, as reported in Fig. 9b. The threshold for $\mathrm{C}$ is close to 11 for this case. Note that, there being no analytical expression for the steady states of the population variables, the threshold must be calculated by integrating the dynamical model to a stationary regime and identifying the curve where extrema disappear.

Globally, our model predicted bistability for $C$ values of about 10-20 [23], within experimental reach, in principle. The cooperativity parameter of the edge-emitting InAs-based structure investigated in Sec. 3 is estimated to be about 5 (with background waveguide losses of $1.5 / \mathrm{cm}$ determined for devices of this kind), i.e. roughly half the requirement for bistability. The analysis of Fig. 11 suggest an optical density of only $\alpha_{0} \approx 14.5 / \mathrm{cm}$ giving $C \approx 3$. The InAlAs-based structure investigated in Sec. 4 is estimated to be about 7 (assuming the same background losses) or about 3 , assuming losses of $10 / \mathrm{cm}$ suggested by the analysis in Sec. 4.2.2. This means all present samples fall short of the requirement for bistability, but an optimization in terms of length and/or an improvement in growth density, number of layers or a reduction in inhomogeneous broadening should open suitable parameter regimes (see also the discussion in Sec. 5).

The determination of the $\mathrm{C}$ parameter required for pattern formation has been discussed in [24] and suggested that QD densities needed (via Eq. 31) for such scopes are not outside reach. More precisely, pattern formation is predicted in the order of $N_{Q D} \approx 10^{12} \mathrm{~cm}^{-2}$ (longitudinally integrated density for vertical-cavity devices) [22].

\section{Experiments on InAs/GaAs quantum dots around $1250 \mathrm{~nm}$}

\subsection{Devices and experimental setup}

The investigated sample is a quantum dot diode (QDD) from Innolume GmbH with a cavity length of $L=1.5 \mathrm{~mm}$. It contains ten layers of InAs QD in a GaAs matrix. The epitaxial structure is similar to the one described in [9]. A single-mode ridgewaveguide ensures spatial fundamental mode operation. The device is designed as a laser with a front reflectivity of $R_{1}=0.18$ and a back reflectivity of $R_{2}=0.99$. The light-current (LI) characteristic is shown in Fig. 10, from which a threshold current of $I=26.6 \mathrm{~mA}$ at $T=15^{\circ} \mathrm{C}$ is inferred. However, in our investigations, it is used only in absorptive mode (i.e. without current injection) or as an amplifier below threshold.

Fig. 10b shows the spontaneous emission spectra taken with an optical spectrum analyzer (OSA, resolution $0.07 \mathrm{~nm}$ ) for three injection current levels. The luminescence is centered around $\lambda=1255 \mathrm{~nm}$. No distinctive peaks or shoulders from excited state or wetting layer emission are visible. The spectrum becomes somewhat jagged around the gain peak at high currents because the finesse of the cavity 

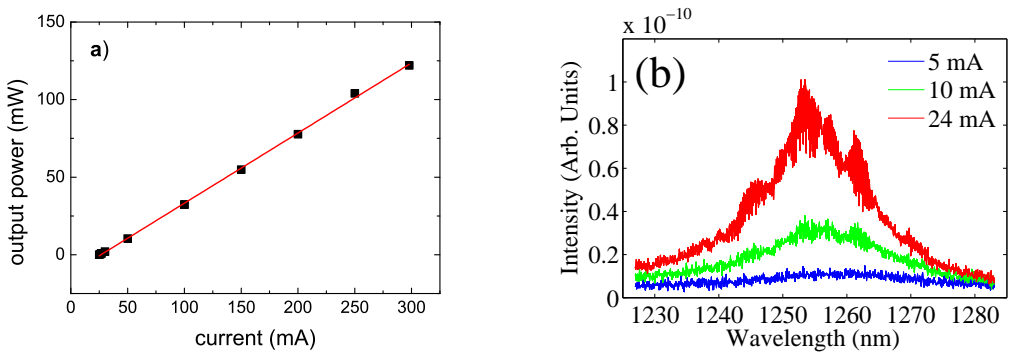

Fig. 10 (Color Online) a) LI-curve (squares) of device with linear fit (straight line), $T=15^{\circ} \mathrm{C}$. b) Spontaneous emission spectra from the QDD for different injection currents below threshold. [Adapted from [41]]

improves. We choose a current of $24 \mathrm{~mA}$ for the detailed investigations under gain conditions.

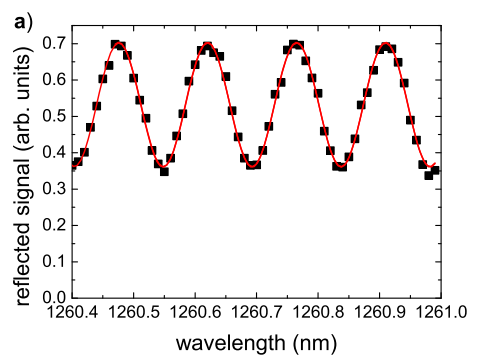

b)

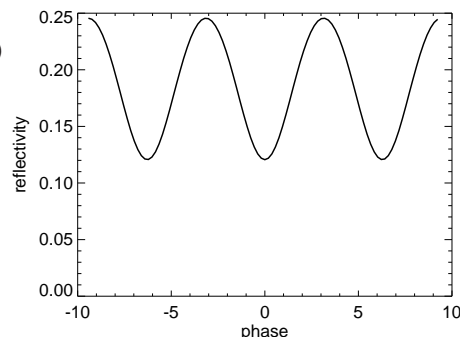

Fig. 11 (Color Online) a) Linear reflection spectrum of QDD (squares) displaying Fabry-Perot fringes and fit to a sine-wave $\left(T=15^{\circ} \mathrm{C}, I=0 \mathrm{~mA}\right)$. b) Calculated Airy-function in reflection for a Fabry-Perot interferometer for $R_{1}=0.18, R=0.99$ and internal (intensity) losses of 16/cm.

Fig. 11a shows a linear reflection spectrum of the QDD obtained from a tunable laser (Santec TSL-210V). The free spectral range is fitted to be $0.15 \mathrm{~nm}$ in agreement with the length specification of the QDD by the manufacturer. The apparent Finesse is about 1. Finesse and modulation depth can be approximately fitted by a modal loss of $16 / \mathrm{cm}$ (see Fig. 10b), somewhat less than the $28.2 / \mathrm{cm}(1.5 / \mathrm{cm}$ waveguide loss and $26.7 / \mathrm{cm}$ absorption) expected from structures of this kind from Sect. 2.2.1, but indicating still a very reasonable interaction strength.

The experimental setup is illustrated in Fig. 12a. The driving laser is a homebuilt Chromium forsterite ( $\mathrm{Cr}$ :Fr) laser [68]. The 4-mirror asymmetric astigmatically compensated $\mathrm{z}$-fold cavity was developed for mode-locked operation but had been re-configured to run in $\mathrm{cw}$ mode with a multi-mode output (see Fig. 12b); the free spectral range of about $100 \mathrm{MHz}$ is obviously not resolved. A prism was included in the laser cavity to allow simple tunability of the system. The output spectra have a full width at half maximum of $0.3 \mathrm{~nm}$ to $0.2 \mathrm{~nm}$, covering several longitudi- 


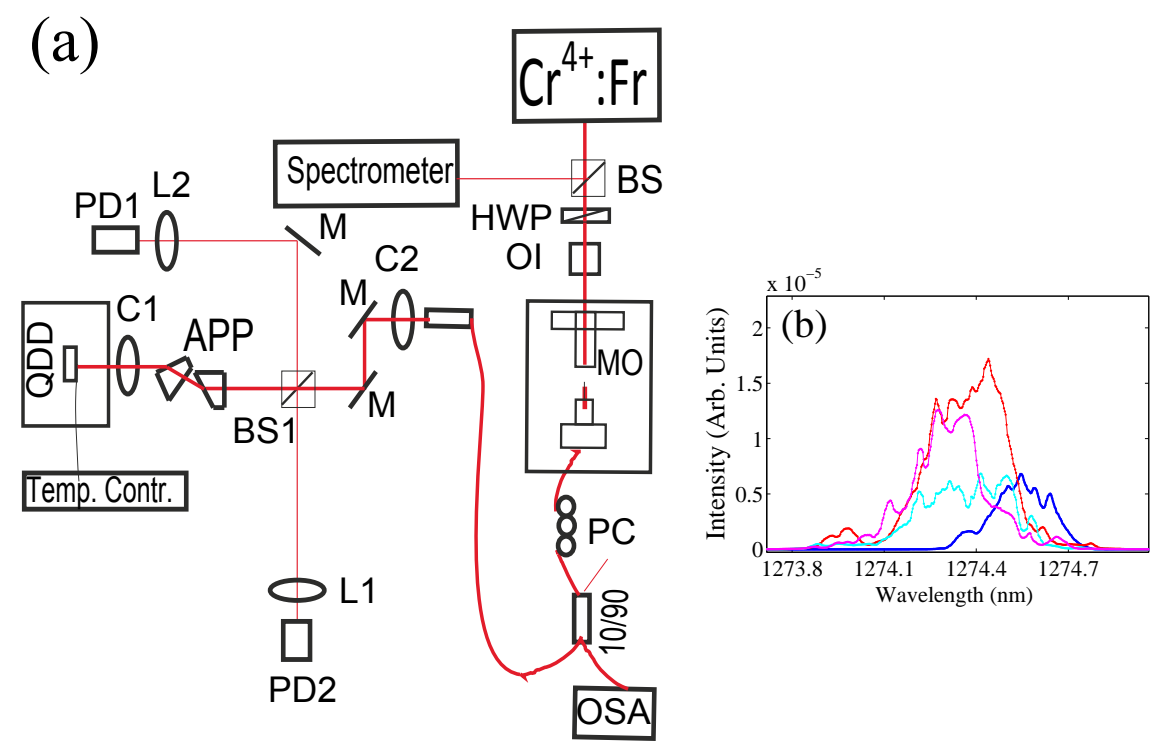

Fig. 12 a) Experimental setup: Chromium forsterite laser $\left(\mathrm{Cr}^{4+}: \mathrm{Fr}\right)$, beam splitter (BS, BS1), halfwave plate (HWP), optical isolator (OI), microscope objective (MO, 40×), optical spectrum analyzer (OSA), mirror (M), anamorphic prism pair (APP), aspherical collimator (C1, $f=3 \mathrm{~mm} ; \mathrm{C} 2$, $f=13.9 \mathrm{~mm}$ ), lenses (L1, $f=50 \mathrm{~mm}$; L2, $f=35 \mathrm{~mm}$ ), amplified photodiode (PD1, PD2). b) Examples for variation of spectra of $\mathrm{Cr}$ :Fr laser over time. [Adapted from [41]]

nal modes of the QDD. Hence, the coherence length of the Cr:Fr laser is less than the QDD cavity length and cavity resonance effects are expected to be weak. Since the laser is not optimized for cw operation, its modal envelope fluctuates slightly over time (some typical examples are shown in Fig. 12b). However, the fluctuations are small against the spectral broadening of the QD, which is on the several $\mathrm{nm}$ to tens of nm level.

After the laser a half-wave plate (HWP) and an optical isolator (OI) are present to avoid back reflection into the laser and for adjusting the power. The beam passing the OI is coupled through a microscope objective (MO) to a single mode fiber. The single mode fiber is then connected to a polarization controller (PC) and to a fiber coupler that splits the incoming beam into $10 \%$ and $90 \%$ components. The weak beam is sent to the OSA to monitor the spectra of the Cr:Fr laser. The main part $(90 \%)$ is collimated by an aspherical lens (C2) with a maximum power of about $100 \mathrm{~mW}$ at $\lambda=(1240-1280) \mathrm{nm}$. An anamorphic prism pair (APP) transforms the circular beam coming out of the fiber to an elliptical beam matching the waveguide mode of the QDD. The PC is used to select the polarization of the beam to be horizontal, matching the dominant polarization of the QDD (TE-polarized). With this scheme we have $100 \mathrm{~mW}$ input power available at the QDD. In principle, further optimization is possible, because the $\mathrm{Cr}$ :Fr laser is capable of producing up to $1 \mathrm{~W}$ in the gain maximum around $1270 \mathrm{~nm}$. 
Then the beam is coupled into the QDD with an aspherical lens (C1). The procedure for the coupling is actually the opposite: The light coming out from the QDD diode is fiber coupled first, with an efficiency of $37 \%$. Then we can conclude that the effective coupling efficiency of the Cr:Fr beam coupled out of the fiber to the QDD waveguide is $c_{e f f}=0.37$. The data given below uses the raw data, i.e. the power incident on the device.

The light reflected by the sample and the light coming out of it is collected via a beam splitter (BS1, Fresnel reflection of 1\% from front surface, back surface ARcoated) and focused onto the output photodiode (PD1). A similar photodiode monitors the input beam as well (PD2). In the experiment, the incident power is varied by turning the half-wave plate and PD1 and PD2 are simultaneously monitored with a digitizer with 16 bit resolution. The reflection coefficient is derived from the ratio of the signal of the two detectors taking the known offsets and sensitivities into account. We plot in the following $R$ (in logarithmic scale) and $\ln R$ (in linear scale) because $\ln R$ should relate to the absorption (gain) coefficient as displayed in Fig. 1 without multi-pass effects, i.e. for a device with perfect anti-reflection coated input facet. Note that in [41] the data were presented as gain (absorption) coefficient per unit length with $L=1.5 \mathrm{~mm}$ in order to facilitate comparison with usual gain coefficients, but we prefer not to do the conversion here due to its strong limitations.

\subsection{Experimental results on saturation of gain and absorption}

Fig. 13(a) shows how the gain coefficient changes as function of input power for $\lambda=1245 \mathrm{~nm}$ under gain, $I=24 \mathrm{~mA}$, and absorptive conditions, $I=0 \mathrm{~mA}$. The reflection coefficient from the QDD shows a pronounced power dependence starting at power levels slightly less than $10 \mathrm{~mW}$ for the absorptive case indicating bleaching of absorption. The reduction of reflectivity due to gain depletion sets in somewhat earlier. Fig. 13(b) shows the corresponding curves for $\lambda=1255 \mathrm{~nm}$ showing similar trends.

The same holds for Fig. 14a displaying the situation for $\lambda=1265 \mathrm{~nm}$ though the small-signal intercepts seem to be slightly reduced. This effect is much stronger in Fig. 14b displaying the data for $\lambda=1280 \mathrm{~nm}$. This is due to the increased detuning to line center. Under gain conditions, there is still a saturation effect, but the absorption is essentially constant (the small decrease at high power is probably an experimental artefact).

The curves in Figs. 13, 14 show the typical behavior of saturation curves, converging at high power to the linear background losses of the structure without active medium, e.g. Fig. 1 in Sec. 2.2.2. Though the effective power within the structure will be affected by multi-pass effects (see below), it seems to be instructive to use the incident powers only for a first analysis because the power within the sample is affected by the saturation level itself in a nontrivial manner. Hence, the dependence of the gain coefficient on power has been fitted with two models that describe saturable absorption in the case of two-level systems with inhomogeneous, Eq. (36), 

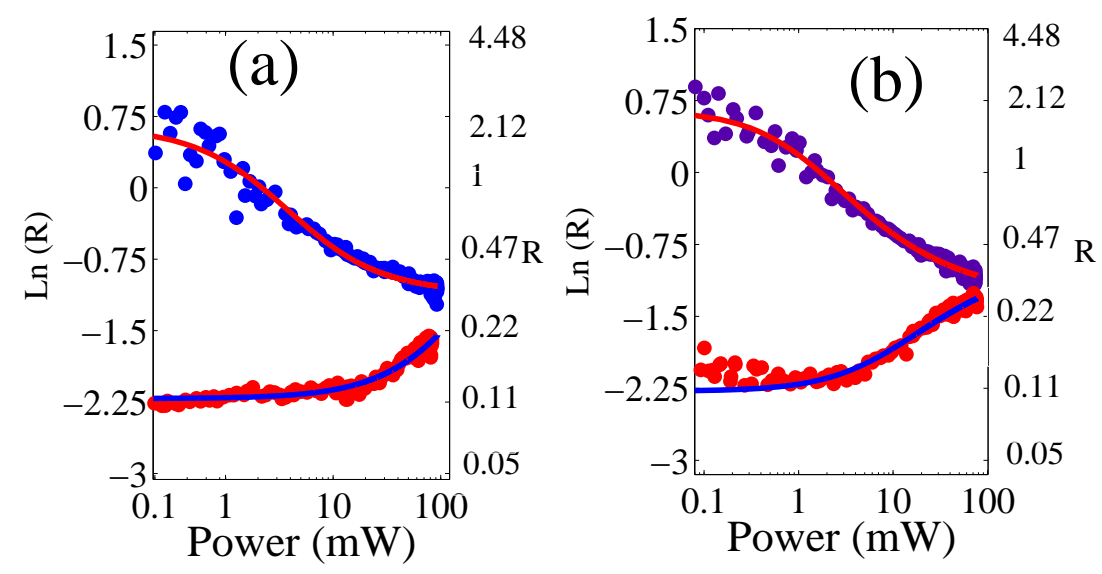

Fig. 13 (Color Online) Reflection coefficient as function of input power [dark gray (blue) data points for $I=24 \mathrm{~mA}$, light gray (red) data points for $I=0 \mathrm{~mA}$ ] and fit to an inhomogeneous broadening model [dark gray (red) line for $I=24 \mathrm{~mA}$, light gray (blue) line for $I=0 \mathrm{~mA}$ ). (a) $\lambda=1245 \mathrm{~nm}$, (b) $\lambda=1255 \mathrm{~nm}$. [Adapted from [41]]

and homogeneous, Eq. (37), broadening. The results shows that both models fit the data at $\lambda=1255 \mathrm{~nm}$ and $\lambda=1265 \mathrm{~nm}$ quite well but the inhomogeneous model proves to fit better and is the only model that fits the data well at $\lambda=1245 \mathrm{~nm}$ and $\lambda=1280$ nm, Fig. 13(a), Fig. 14(b).

Fig. 15 shows the saturation power under absorptive and gain conditions as function of wavelength as obtained from the fits displayed in Figs. 13, 14. The minimum saturation power is reached at $\lambda=1255 \mathrm{~nm}$, i.e. line center, as expected, and is $P_{\text {sat }}=9 \mathrm{~mW}$ in the absorptive case and $P_{s a t}=1.4 \mathrm{~mW}$ in the gain case. The saturation power increases for increasing detuning. These tendencies are in qualitative agreement with the simulations presented in Sect. 2.3.

\subsection{Analysis and discussion}

Via $I_{\text {sat }}=2 P_{\text {sat }} c_{\text {eff }} /\left(\pi \mathrm{w}_{x} \mathrm{w}_{y}\right)$, where $\mathrm{P}_{\text {sat }}$ are the saturation powers reported in Fig. 15 and $w_{x}=3 \times 10^{-6} \mathrm{~m}$ and $w_{y}=0.5 \times 10^{-6} \mathrm{~m}$ are the beam radii in the slow, respectively fast, axis, a saturation intensity in line center of $I_{\text {sat }}=1.4 \times 10^{9} \mathrm{~W} / \mathrm{m}^{2}$ 


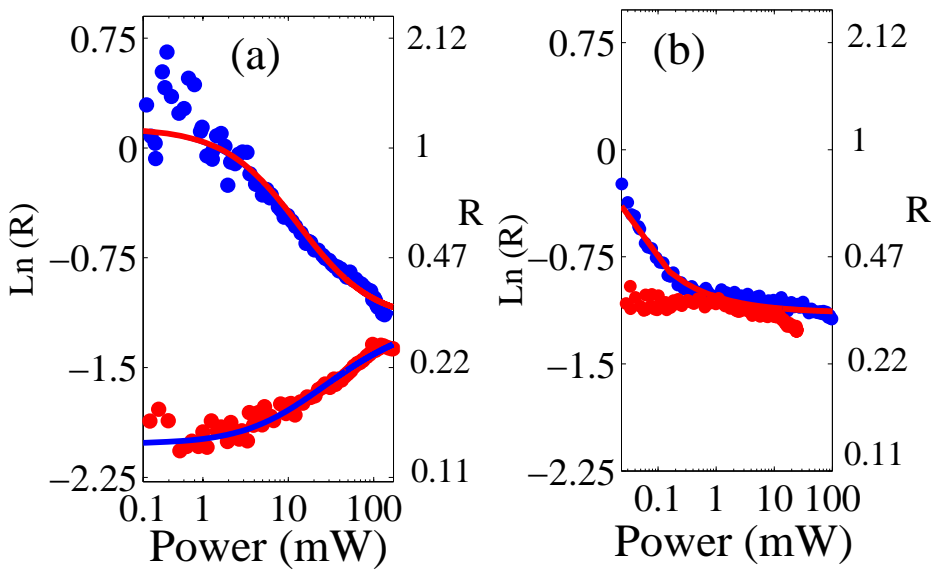

Fig. 14 (Color Online) Gain coefficient as function of input power at (a) $\lambda=1265 \mathrm{~nm}$, (b) $\lambda=1280 \mathrm{~nm}$. Legend as in Fig. 13. [Adapted from [41]]

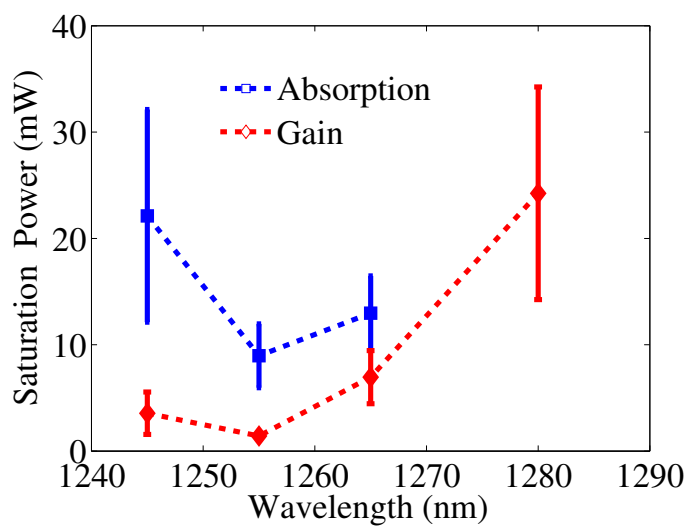

Fig. 15 (Color Online) Saturation power as function of wavelength in absorption and gain cases. [Adapted from [41]]

is obtained for the absorptive case. This value agrees with numerical predictions using the parameters from Sect. 2.3 and Eq. 22 for a carrier lifetime in the ground state of 60 ps, i.e. a rather small value.

A qualitative difference between the experimental results and the simulations is that in the simulations the absorption saturates earlier than the gain, i.e. the theoretical expectation for the gain case based on Sect. 2.3 is $I_{\text {sat }}=2.8 \times 10^{9} \mathrm{~W} / \mathrm{m}^{2}$, whereas the fit from the experiments yields $I_{\text {sat }}=0.2 \times 10^{9} \mathrm{~W} / \mathrm{m}^{2}$. 
This feature, as well as the absolute scaling of the reflection coefficients, is strongly influenced by the fact that the sample does not have AR-coatings on both ends but the coatings are designed for laser operation. As indicated, we don't expect strong cavity resonance effects because of the low coherence length of the input laser, but nevertheless the device will behave as a two-pass amplifier due to the high reflectivity of the back mirror. Some portion of the light attenuated or amplified after one double pass $2 L$ will be in turn retro-reflected into the sample due to the finite reflectivity of the front facet. The expected total reflectivity can be estimated from the incoherent sum of intensities of the multi-pass configuration,

$$
R=R_{1}+c_{\text {eff }}\left(1-R_{1}\right)^{2} \frac{R_{2} e^{\left(-\alpha_{g}+g\right) 2 L}}{1-R_{1} R_{2} e^{\left(-\alpha_{g}+g\right) 2 L}}
$$

where $\alpha_{g} \approx 1.5 / \mathrm{cm}$ represents the waveguide loss and $g=-\alpha$ is the modal gain coefficient. (Qualitatively similar results are obtained if one averages the coherent Airy function over the resonator phase.) The corresponding function is displayed in Fig. 16 as the curved solid curve.

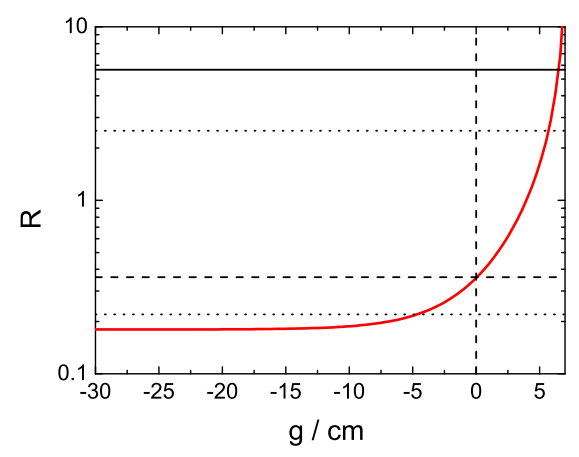

Fig. 16 (Color Online) Reflection coefficient calculated from Eq. (45) versus modal gain coefficient [Light gray (red) solid line]. Dashed lines: Value for high power asymptote $(g=0)$. Solid black line: Estimated maximal linear gain. Dotted lines: Reduction of linear absorption and gain by factor of $\sqrt{2}$.

Obviously, the small-signal coefficient in the absorptive case $(g \approx-27 / \mathrm{cm})$ is totally determined by the reflectivity $R_{1} \approx 0.18$ of the front facet. This is in rough agreement with the experiment assuming some additional linear losses. The reflection coefficient for the completely bleached QD, $g=0 / \mathrm{cm}$, is about 0.36 (dashed black lines in Fig. 16) in rough agreement with the high power asymptotes. Finally, the threshold gain for the laser structure can be estimated to be $7.25 / \mathrm{cm}$. Assuming a gain of $6.5 / \mathrm{cm}$ for the just below threshold case analyzed experimentally, the reflection coefficient is about 5.7 (solid black line in Fig. 16), again in rough agreement with the experiment assuming some additional linear losses. These multi-pass 
effects explain also the difference in saturation power between gain and absorption: A change of modal gain from $6.5 / \mathrm{cm}$ to only $5.7 / \mathrm{cm}$ is required to change the externally observed reflection coefficient by a factor of $\sqrt{2}$, whereas the modal absorption needs to change from $-27 / \mathrm{cm}$ to $-4.6 / \mathrm{cm}$ to achieve the same effect. This is easy to understand hence absorption will reduce the intensity propagation further down into the structure and thus hinder saturation. In contrast, with gain the intensity increases with propagation in the structure and hence saturation becomes easier. Hence, the multi-pass effects delay saturation in absorption and favor it in gain explaining the observed asymmetry.

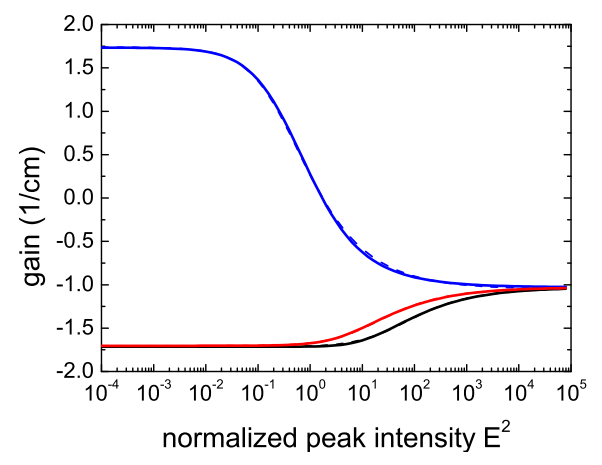

Fig. 17 (Color Online) Reflection coefficient calculated from Eqs. (45), (36) versus normalized intensity. Black line: $g(0)=-27 / \mathrm{cm}$, red (light gray) line: $g(0)=-14.5 / \mathrm{cm}$, blue (dark grey) line: $g(0)=+6.5 / \mathrm{cm}$. Dashed lines denote fits of these plots to the saturation law (36) and are essentially not distinguishable from the data.

One can now use the saturation law (36) to supply the intensity dependence of $g$ in (45). The resulting reflection coefficient is displayed in Fig. 17 for different small signal absorption and gain values. It is apparent that the curves have a qualitative similarity to the experimental curves in Fig. 13. Furthermore, they can be essentially perfectly fitted by the saturation law (36). From the fit one obtains $E_{s}^{2}=0.29$ for the gain case, $g(0)=+6.5 / \mathrm{cm}$, and $E_{s}^{2}=33.3$ for the absorptive case, $g(0)=-27 / \mathrm{cm}$. This confirms the asymmetry between saturation of absorption and gain discussed above, but the ratio between these values is very high, about 110 , whereas the experimentally observed ratio is significantly smaller, around 7. It turns out that these values are very sensitive to the total absorption, e.g. for $g(0)=-14.5 / \mathrm{cm}$ (inferred earlier from Fig. 11) $E_{s}^{2}=9.6$. This is reasonable since the depletion of the pump and hence the delay of saturation - will be stronger, if the linear absorption coefficient is stronger. For this value of $g(0)$ the ratio between the saturation intensity of gain and absorption is about 30, still larger than in the experiment. In view of the uncertainties, one cannot make strong statements but it appears that the 'material' (i.e. not influenced by multiple-pass effects) saturation intensity is about $5 \times 10^{8} \mathrm{~W} / \mathrm{m}^{2}$, in between the values observed for the gain and the absorption case. This would 
correspond to a lifetime of the GS of $170 \mathrm{ps,} \mathrm{which} \mathrm{is} \mathrm{a} \mathrm{low} \mathrm{though} \mathrm{still} \mathrm{reasonable}$ value.

For SESAM applications, saturation fluences for 1280-1340 nm QD under shortpulse excitation are reported to be $0.02-0.25 \mathrm{~J} / \mathrm{m}^{2}[8,7,18]$. If the carrier decay within the width of the probing pulse can be neglected, the saturation fluence can be converted to an equivalent $\mathrm{cw}$ saturation intensity by multiplying it with the carrier decay rate. Hence for a lifetime of 100 ps one concludes on saturation intensities of $(0.2-2.5) \times 10^{9} \mathrm{~W} / \mathrm{m}^{2}$ in line with our observation.

\subsection{Experiments addressing nonlinear index shifts}

As indicated from the discussion above, the data for the saturation of the imaginary part of the refractive index (i.e. gain or absorption) would be much cleaner and more straightforward to analyze if the sample would have been AR-coated at both input facets (or the waveguide would have been tilted with respect to the facets). The choice for a laser samples instead of an AR-coated amplifier stemmed from the desire to probe nonlinear index shifts via the shift of Fabry-Perot fringes as explained in Sect. 2.3.1.

We briefly describe the experiment. A low-amplitude beam of a tunable laser was injected into the other input of the 90:10 fiber splitter. It is scanned over 0.5 nm, i.e. several FSR of the QDD, and its reflection is measured in presence of the strong pump laser. The tunable probe beam is chopped at a frequency of $800 \mathrm{~Hz}$ and its weak signal is filtered out of the total reflection signal with the help of a lock-in amplifier. We observe a change in shape and Finesse of the Airy function, i.e. a nonlinear version of Fig. 11a, dependent on the pump power, which is qualitatively as expected. This indicates that the idea of the measurement works in principle. However, the shift is about $0.001 \mathrm{~nm} / \mathrm{mW}$ (external power) independent of wavelength and current. As one expect a different sign of the shift under absorptive and gain conditions, we conclude that we don't probe a carrier effect but some background absorption. For comparison, the fringe shift with temperature was determined to be $0.1 \mathrm{~nm} / \mathrm{K}$, i.e. the effect can be caused by very small temperature variations. The origin of this shift is unclear at the moment.

\section{Experiments on InAlAs/GaAlAs quantum dots at $780 \mathrm{~nm}$}

While QD have been developed first in the InAs/GaAs [69] system which operates typically at wavelengths larger than $1 \mu \mathrm{m}$, the question of connecting with wavelength domains compatible with the GaAlAs material system and with Ti:Sa lasers is actually being raised. InAs/GaAs QD can obviously not provide the solution since they lead to very shallow dots improper to the proposed target of realizing quasi 2-level electronic systems in this spectral range. Increasing the barrier 
height may be obtained by evenly incorporating aluminium in both the barrier and the dot materials leading to a higher confinement of quantum states and to the forsaken spectral overlap with the GaAlAs system. In this framework, InAlAs/GaAlAs QD [70, 71] open very promising perspectives both for $\mathrm{cw}$ and pulsed nonlinear or even self-organizing optical systems. In this section, structural and optical properties of MBE-grown InAlAs/GaAlAs quantum dots are investigated as a function of the growth kinetic and thermodynamical conditions. Appropriate choice of growth conditions allows to control the density as well as the average size of QD. This establishes also an excellent approach for controlling the carrier lifetimes during the growth stage, and introduces means of realizing either a slow focusing Kerr effect (FKE) material (ns-scale) or a fast (ps-scale) saturable absorber, as started in [12].

In this section, we report on linear and nonlinear measurements of the susceptibility of InAlAs/GaAlAs QD, after describing their growth conditions, which correlate to their structural properties in terms of dot and dislocation densities. Optical quality is established through spectral and temporal photoluminescence (PL) measurements. The core of the section is dedicated to the measurement of group index and nonlinear absorption using a long (high-order) Fabry-Perot cavity embedding InAlAs/GaAlAs QD. This approach leads to unprecedented measurements for this material and establishes, though not yet fully exploited, quite an efficient saturation behavior.

\subsection{Description of the experiments}

\subsubsection{Material properties and device structure}

The sample was MBE-grown on a GaAs substrate, using the Stransky-Krastanov growth regime. According to the criteria developed in the model, Sec. 2.1, there is a necessity to obtain a large volume of interaction between QDs and light by increasing the overall density of dots, and thereby the sensitivity of our experimental setup to the QD contribution to the susceptibility. This was found in growing five superimposed planes of $\mathrm{In}_{0.67} \mathrm{Al}_{0.33}$ As QDs with $\mathrm{Ga}_{0.67} \mathrm{Al}_{0.33}$ As barriers as the nonlinear material (Fig. 18a). The symmetric step-graded barrier and cladding layers with corresponding material concentration follow and a GaAs cap terminates the structure. A cross-sectional visualization of QD was performed that gave information on the relaxation degree of the QDs which revealed appropriate for optical experiments. Due to the depth at which QD layers are located with respect to the surface, the density of dots $\rho_{D}$ could only be inferred from a cross-sectional TEM image and lead to an integrated density for the 5 layers such as $2 \times 10^{11}<\rho_{D}<5 \times 10^{11} \mathrm{~cm}^{-2}$. Onelayer samples grown under nominally the same growth conditions had a density of about $2 \times 10^{11} \mathrm{~cm}^{-2}$.

The photoluminescence spectrum of this sample is sketched in Fig. 19. It exhibits a $40 \mathrm{~nm}$ wide inhomogeneous peak around $780 \mathrm{~nm}$ well separated from the other transitions with the $\operatorname{In}_{0.67} \mathrm{Al}_{0.33} \mathrm{As} \mathrm{WL}$ and the $\mathrm{GaAl}_{0.33}$ As barrier. 
a)

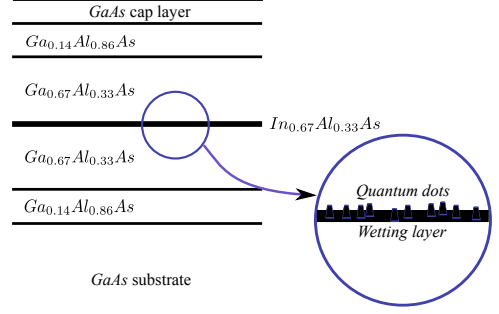

b)

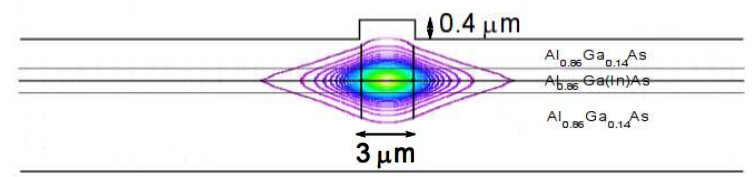

Fig. 18 a) 5-layer AlGaAs/AlInAs QD structure. b) Ridge waveguide formed by the initial stack modified for waveguiding properties (see text). The single mode size calculated with a finite element program (ALCOR) is $3.0 \times 0.250 \mu \mathrm{m}^{2}$. [Adapted from [42]]

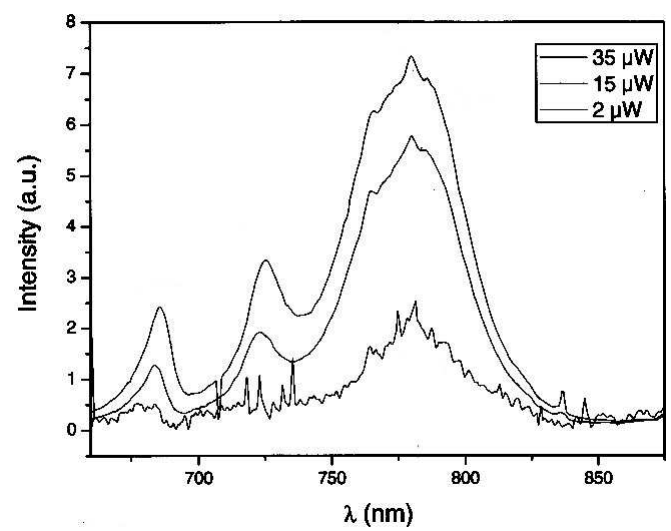

Fig. 19 Photoluminescence spectrum of the InAlAs/GaAlAs QD used in the experiment at different excitation levels.

The complete structure was designed so as to form a planar waveguide inducing a vertical optical confinement (Fig. 18 a and b). It comprises two $1.5 \mu \mathrm{m}$-thick $\mathrm{Ga}_{0.14} \mathrm{Al}_{0.86} \mathrm{As}$ cladding layers sandwiching the core layer consisting of the five layers of $\mathrm{In}_{0.67} \mathrm{Al}_{0.33} \mathrm{As}$ QDs spaced by $41.7 \mathrm{~nm}$-thick $\mathrm{Ga}_{0.67} \mathrm{Al}_{0.33} \mathrm{As}$ barrier spacers. Finally, lateral optical confinement is introduced by etching the surface with a $0.4 \mu \mathrm{m}$ deep, $3 \mu \mathrm{m}$ wide ridge. The values were retained on the basis of finite element optical modeling with the finite element program (C)ALCOR warranting the monomode character of this waveguide around $800 \mathrm{~nm}$. The optical mode area at half-intensity is $3 \mu \mathrm{m} \times 0.250 \mu \mathrm{m}$ (Fig. 18b). 


\subsubsection{Principle of the experiment}

In contrast to Sec. 3, the driving TiSa-laser is a highly coherent single-frequency laser and hence interference effects in the Fabry-Perot sample investigated need to be taken into account. The measurements rely on the use of the technique described in Sec. 2.2.2 and 2.3.2 with $R=0.30$ reflectivity mirrors provided by the uncoated cleaved facets of the sample.

In the presence of absorption with coefficient $\alpha$, one uses the transmittance $T$ defined as the ratio of the transmitted $\left(I_{t}\right)$ to incident $\left(I_{i}\right)$ intensities [72, 73]

$$
T=\frac{(1-R)^{2} e^{-\alpha L}}{\left(1-R e^{-\alpha L}\right)^{2}+4 R e^{-\alpha L} \sin ^{2}(\phi)},
$$

where $\Phi$ is the phase accumulated during the propagation of light inside the cavity of length $L$. If it is filled with a material of intensity-dependent refractive index $n(I)$ the single pass phase accumulated is

$$
\phi \equiv \phi(I, L)=\int_{0}^{L} \frac{2 \pi n(I(z))}{\lambda} d z .
$$

We neglect the eventual dependence of the intracavity intensity on the position and use instead the longitudinal average intensity $\bar{I}=\frac{1}{L} \int_{0}^{L} I(z) d z . T$ is thus a function of the intracavity intensity via $\phi$ and $\alpha$. Similarly the incident intensity $I_{i}$ can be connected to $\bar{I}$ via [73]

$$
I_{i}=\frac{\alpha L}{(1-R)\left(1+R e^{-\alpha L}\right)\left(1-e^{-\alpha L}\right)}\left[\left(1-\operatorname{Re}^{-\alpha L}\right)^{2}+4 \operatorname{Re}^{-\alpha L} \sin ^{2}(\phi)\right] \bar{I} .
$$

From this point we shall drop the bar and write the average intensity as $I$. The general resolution for arbitrary and eventually nonlinear $\phi$ 's may be found in solving Eq. 46 for $A=e^{-\alpha(I) L}$. Defining $S(I, \lambda) \equiv \sin ^{2}(\phi)$ this leads to the equation

$$
R^{2} T A^{2}+\left[2 R T(2 S-1)-(1-R)^{2}\right] A+T=0
$$

with the minus signed retained solution

$$
A=\frac{-\beta-\sqrt{\beta^{2}-4 R^{2} T^{2}}}{2 R^{2} T},
$$

where $\beta(I, \lambda)$ is the coefficient of $A$ in the second order Eq. 49. This gives in turn an experimental access to the nonlinear coefficients as the absorption coefficient comes straightforwardly as $\alpha=-\ln A / L$ and can be deduced from the knowledge of the transmission $T$. In turn, the nonlinear index determination is not straightforward and can only be accessed through the determination of the phase as an adjustable parameter. However in the present case, the QD are supposed to have a saturating absorption, 


$$
\alpha(I)=\alpha_{0} \frac{I_{s}}{I+I_{s}}
$$

and a saturating index

$$
n(I)=n_{b}-\delta n_{s} \frac{I_{s}}{I+I_{s}} .
$$

This corresponds to the theory developed in Sec. 2.1, however assuming strong homogeneous broadening for simplicity. Eq. 50 reads as an implicit equation in $A$ where the RHS of Eq. 50 depends on the transmission $T$ and on $A$ via $\beta$. This derivation of optical coefficients from experimental transmission measurements can be obtained via numerical or converging fit techniques.

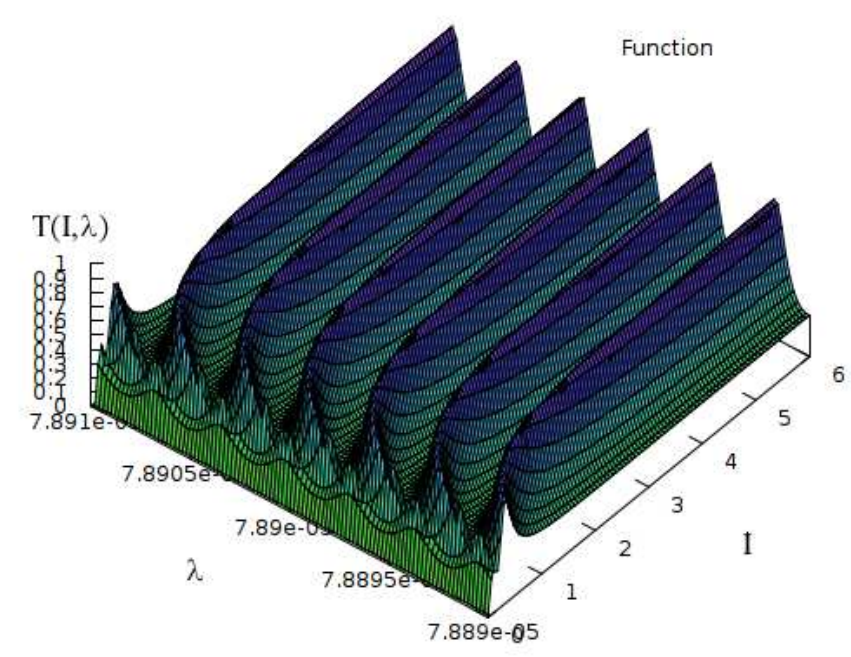

Fig. 20 Calculation of the transmission of nonlinear saturating Fabry-Perot resonator. The data used are $: R=0.3, \Gamma_{\text {trans }} \alpha_{0}=14 \mathrm{~cm}^{-1}, \alpha_{g}=10 \mathrm{~cm}^{-1}, L=0.23 \mathrm{~cm}, I_{s}=2.8 \mathrm{~kW} / \mathrm{cm}^{2}, \Gamma_{\text {trans }} \delta n_{s}=$ $1.75 \times 10^{-3}$. $I$ is scaled to the saturation intensity.

The transmitted intensity $I_{t}$ is known to be directly related to the intracavity field intensity $I$

$$
I_{t}=\frac{\alpha L e^{-\alpha L}(1-R)}{\left(1+R e^{-\alpha L}\right)\left(1-e^{-\alpha L}\right)} I .
$$

Under the effect of the real and imaginary parts of the nonlinear susceptibility, both the transmission spectrum - via $\phi-$ and the transmission amplitude maximum - via saturation - are modulated. The combined measurement of $I_{t}$ and $I_{i}$ as a function of the wavenumber (wavelength) gives thus access to the transmittance and to 
the intracavity intensity. In the case of a waveguide, we use also the transverse confinement factor $\Gamma_{\text {trans }}$ which weighs the QD layer contribution to the nonlinear index with respect to the mode area. As a general formulation, $n(I) \equiv n_{0}+\Gamma_{\text {trans }} \delta n(I)$, should the dependence on $I$ be linear or saturating.

The five InAlAs QD layers have a total thickness of approximately $5 \times 64 \mathrm{~nm}=$ $30 \mathrm{~nm}$. As they only partially overlap with the mode transverse dimension $250 \mathrm{~nm}$ we deduce a transverse confinement factor $\Gamma_{\text {trans }} \simeq 0.12$. Bulk and MQW semiconductors are known to exhibit nonlinear index saturation of the order of $10^{-2}$ [74]. Under these assumptions (other parameters in caption of Fig. 20) one can calculate now the expected transmission characteristics of the nonlinear Fabry-Perot interferometer in dependence on wavelength and intensity. Fig. 20 shows the characteristic ripple structure of the transmission of a Fabry-Perot cavity. The modulation depths increases with intensity due to the increase of Finesse with saturation of absorption. The fringes shift due to the nonlinear index change. The figure confirms the backof-the envelope calculation in Sec. 2.3.2 that phase shifts of some $10^{-4}$ should be detectable in mm-sized cavities due to the high interference order.

\subsubsection{Experimental setup}

The experimental setup, similar to that used in Sec. 3 uses a Ti-Sa laser source operating in the short wavelength range between $760 \mathrm{~nm}$ and $810 \mathrm{~nm}$ and emitting 100-500 mW cw power. A small fraction is collected via a beamsplitter (BS) into a Burleigh WA1100 wavemeter. In order to prevent thermal drifts of the sample properties, the beam intensity is modulated by an acousto-optic modulator (AOM) in the deflection mode into rectangular or triangular envelopes with a $10 \mathrm{kHz}$ frequency and a duty cycle ranging from 10 to 20, converted from an electrical signal produced by an ANALOGIC 2000 arbitrary function generator. The modulated beam is then injected into a monomode fiber and directed to the monomode waveguide containing the QDs. In the path, light intensity is adjusted by a polarization controller injecting a 10/90 directional coupler whose $10 \%$ branch allows the measurement of a signal proportional to the incident power onto the signal on Photodiode PD1. The 90\% branch is coupled via the lensed end of a tapered fibre into the waveguide and its output, collected by a microscope objective (L6), is detected by a second photodiode PD2.

The sample is mounted on a Peltier element holder giving a temperature control better than $0.1 \mathrm{~K}$. The alignment procedure is performed with the help of two cameras. Camera 1 allows the precise pre-positioning of the fiber with respect to the front facet of the waveguide and Camera 2 allows the visualization of the rear facet and the optimization of the optical injection. Finally, three parameters could be measured: $I_{i}, I_{t}$ and $\lambda$. In practice, the measurement sequence consisted in ramping $I_{i}$ for a given $\lambda$ and measuring $I_{t}$, then deducing the intracavity intensity $I$ through Eq. 53 and finally recording and plotting the $3 D$ curve

$$
T \equiv T(I, \lambda)
$$




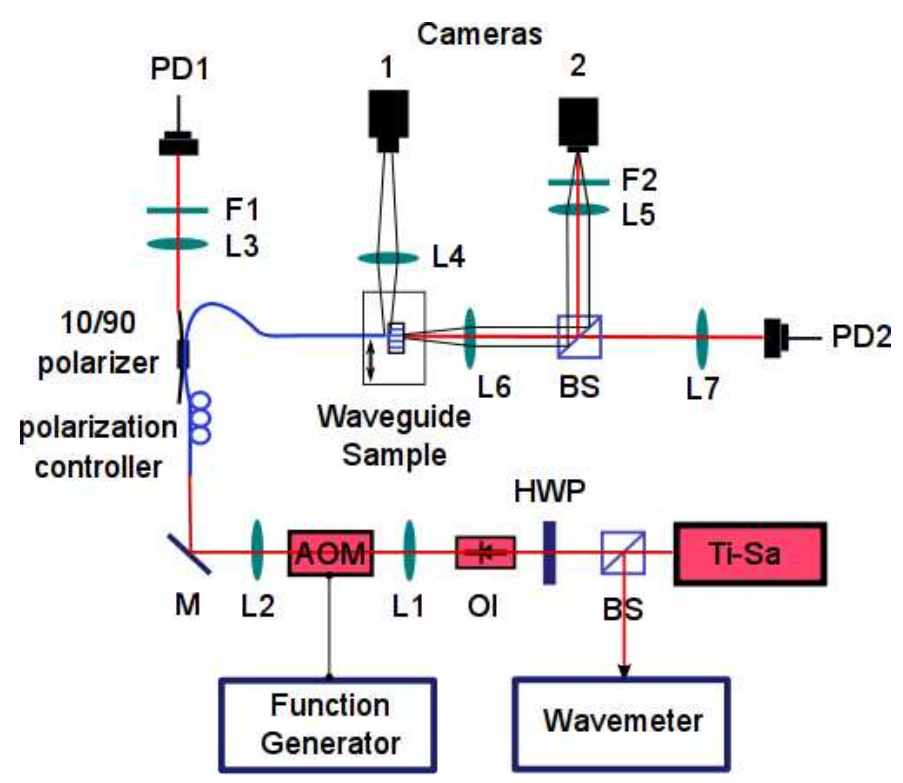

Fig. 21 Experimental setup: Ti:Sapphire laser (Ti-Sa), beam splitter (BS), half-wave plate (HWP), optical isolator (OI), acousto-optic modulator (AOM), lenses L1 $(f=10 \mathrm{~mm}), \mathrm{L} 2(f=10 \mathrm{~mm})$, L3 $(f=35 \mathrm{~mm}), \mathrm{L} 4(f=50 \mathrm{~mm}), \mathrm{L} 5(f=35 \mathrm{~mm}), \mathrm{L} 6(f=35 \mathrm{~mm}), \mathrm{L} 7(f=35 \mathrm{~mm})$; mirror (M), filters (F1,F2), photodiodes (PD1,PD2). [From [42]]

\subsection{Experimental results on saturation of absorption}

\subsubsection{Group index measurement}

A first series of measurements was performed in order to detect the nonlinear index variation under increasing illumination. The waveguide length is $L=0.23 \mathrm{~cm}$. Incident and transmitted intensities were recorded by ramping the incident intensity through the AOM at a fixed wavelength and iterating with $1 \mathrm{pm}$ wavelength steps over a $14 \mathrm{pm}$ range around the central wavelength. Such recordings were performed around $765 \mathrm{~nm}, 780 \mathrm{~nm}, 787 \mathrm{~nm}, 790 \mathrm{~nm}, 795 \mathrm{~nm}$, and $800 \mathrm{~nm}$ in order to scan different detunings from the inhomogeneously broadened line. Regular modulation are displayed at wavelengths around $\lambda=787 \mathrm{~nm}$ (Fig. 22a).

At low intensities, it was not possible to observe any drift of the Fabry-Perot resonances under increasing illumination while at higher intensities, absorption saturation restores the phase modulation, qualitatively as in Fig. 20 discussed above, but with a contrast poorer than expected. Both were attributed to the existence of a non saturable part of the absorption $\left(\alpha_{g}\right)$ that probably enforces the damping of the intracavity interferences at low intensities and maintain the cavity at a finesse lower than that of the cold cavity. This is confirmed by calculations (Fig. 22b) where absorption was modified as 
a)

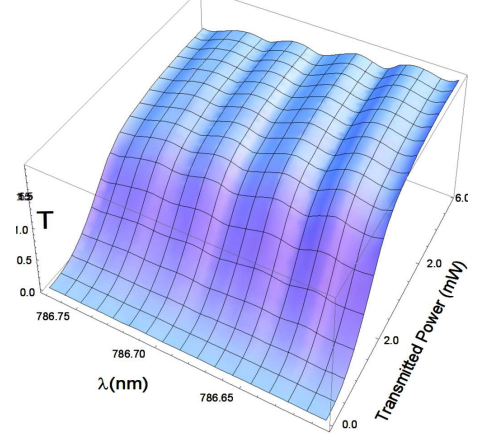

b)

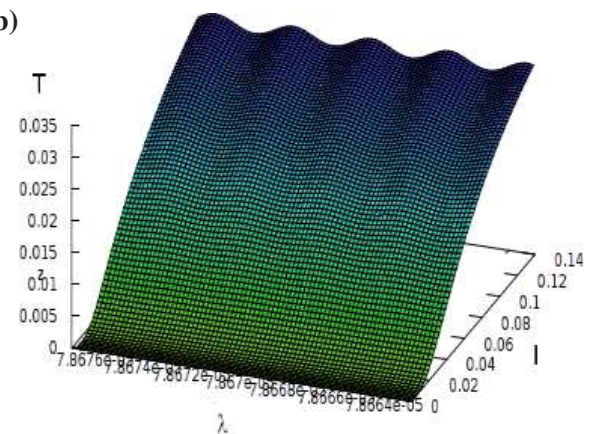

Fig. 22 a) Plot of the transmission as function of wavelength for different transmitted intensities; b) Theoretical plot assuming the fitting parameters obtained including nonsaturable losses as input parameters. [From [42]]

$$
\alpha(I)=\alpha_{g}+\alpha_{0} \frac{I_{s}}{I+I_{s}}
$$

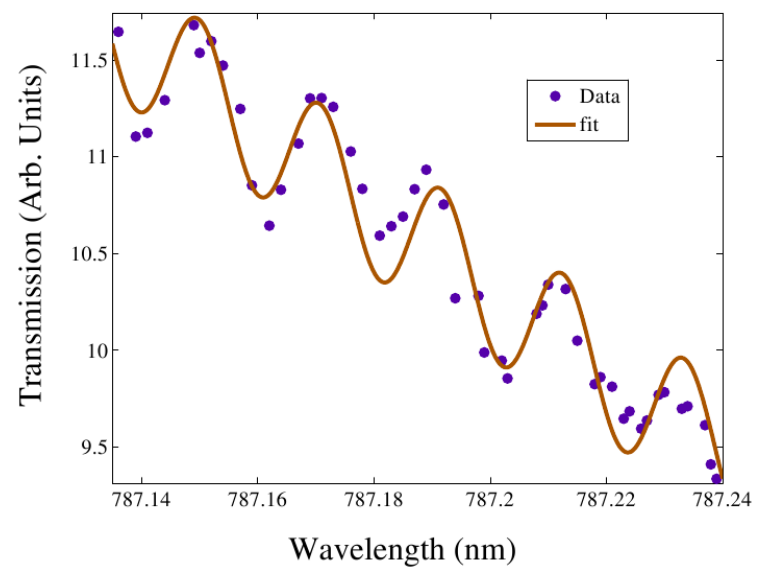

Fig. 23 Transmission spectrum at high intensities with saturated absorption at constant intracavity intensity around $787 \mathrm{~nm}$. [From [42]]

From data at high transmission (see Fig. 23), a rather precise measurement of the free spectral range as $15.6 \mathrm{GHz}$ or $0.52 \mathrm{~cm}^{-1}$ wavenumbers allowed to calculate the group index, incorporating the modal and spectral contributions to dispersion. This leads to the group index value $n_{g}=4.16$. This is in good agreement with the literature value of 4.12 for the group index of $\mathrm{Al}_{0.28} \mathrm{Ga}_{0.72} \mathrm{As}$ at $787 \mathrm{~nm}$ [75] allowing for waveguide dispersion.

In view of the fact that the measurement was done close to the absorption maximum, where the phase shifts are small (e.g. Figs. 5, 7) it is consistent that no shift 
of the Fabry-Perot fringes was detectable. Further investigations need to be focused on higher detunings to probe for phase shifts.

\subsubsection{Absorption saturation}

As Fig. 22 indicated already, the experimental setup can be used to measure the absorption of the quantum dots embedded in the waveguide section. The wavelength $\lambda=789.00 \mathrm{~nm}$ is chosen so as to lie rather close to the maximum of QD absorption line. Transmission measurements as expressed by Eq. 50 give indeed access to absorption. This was done as previously by ramping the incident intensity and measuring the transmittance. Fig. 24a shows the transmission as a function of the incident intensity. Fig. 24b plots the absorption as deduced from Eq. 50 applied to the experimental data and fitted with the ansatz of Eq. 51. The difficulty lies in the dependance of $T$ upon the nonlinear index via $\phi$. Therefore, instead of using the intracavity intensity, which depends on this phase term, we used the measured incident intensity with the assumption that they are in a quasi constant ratio. We first adjusted the saturation parameters of the absorption in Eq. 24 so as to obtain the best possible fit. However, we noted that in a second step additional adjustments can be made by fine tuning the wavelength i.e the position with respect to the resonance and the saturation index. Further experiments will attempt to elucidate this point. However, from these adjusted values, we could extract the significant parameters of the QD material.

a)

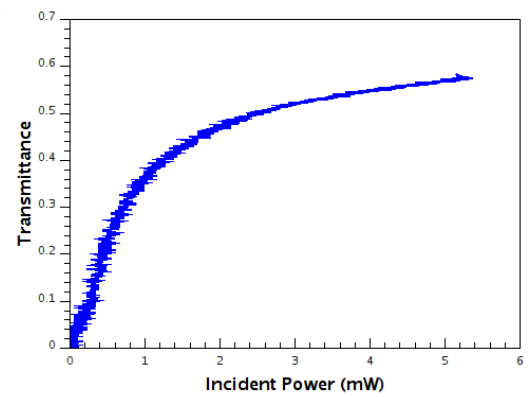

b)

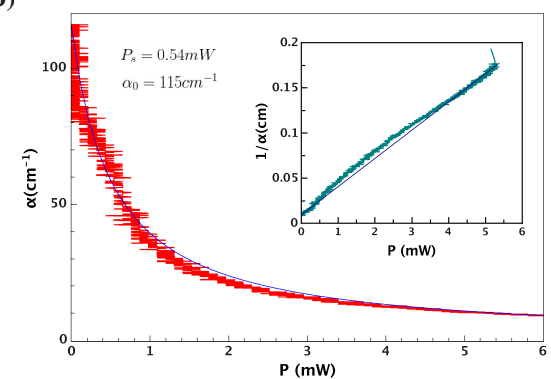

Fig. 24 a) Plot of the transmission as a function of the incident intensity. b) Material absorption vs incident intensity as deduced from Eq. 50, red (experimental), black (theoretical). The inset displays the same data in the form $1 / \alpha=f\left(P_{\text {inc }}\right)$ the linear dependence assesses a saturation law of the absorption. [Adapted from [42]]

The calibration of the intracavity power was performed by using its relationship (Eq. 48) with incident power $\left(P_{\max }^{i n}=5.2 \mathrm{~mW}\right.$ ). In addition we introduced the coupling coefficient of light into the waveguide $\gamma=0.083$ defined as the overlap ratio of the focused beam area $3 \times 3 \mu \mathrm{m}^{2}$ to that of the mode $S=0.250 \times 3 \mu \mathrm{m}^{2}$. The maximum intracavity power is therefore $P_{\max } \simeq 0.54 \mathrm{~mW}$. 
The absorption curve appears indeed to have a saturation behavior leading to a vanishing absorption coefficient at high intensities. In Fig. 24b two curves are presented for $\alpha$ and for $1 / \alpha$ in the inset calculated with Eq. 50 and fitted according to a saturation ansatz also corrected with the transverse confinement factor $\Gamma_{\text {trans }}$

$$
\alpha_{\text {measured }}(I)=\alpha_{g}+\Gamma_{\text {trans }} \alpha_{0} \frac{P_{s}}{P+P_{s}},
$$

where we used $\alpha_{g}=10 \mathrm{~cm}^{-1}$, as suggested by the analysis of Fig. 22. They yield an unsaturated absorption value of $\Gamma_{\text {trans }} \alpha_{0}=13.8 \mathrm{~cm}^{-1}, \alpha_{0}=115 \mathrm{~cm}^{-1}$ and a saturation power $P_{S} \simeq 0.021 \mathrm{~mW}$ with excellent fidelity. The deduced saturation intensity is

$$
I_{S}=\frac{P_{s}}{S}=2.8 \mathrm{~kW} / \mathrm{cm}^{2} .
$$

It could be interesting to compare this value with those obtained in other experiments. However the comparison must be performed accounting with the experimental conditions at which they were performed, cw or pulsed. In the cw regime, QD are expected in general to have a lower saturation intensity than material of lower confinement dimensionality. In [74] for example, a series of measurements were performed on bulk and MQW Urbach's tail, yielding a saturation intensity of $1-3 \times 10^{5} \mathrm{~W} / \mathrm{cm}^{2}$ depending on detuning. In [74] it is argued also that - due to the detuning dependence - the relevant parameter for comparison is the factor $\alpha_{0} I_{s}$ proportional to the transparency carrier density. We obtain here $\alpha_{0} I_{s} \simeq 3 \times 10^{5} \mathrm{Wcm}^{-3}$ which confirm the reduction of the saturation parameters in InAlAs/GaAlAs QDs whose values were respectively in the range $0.1-1 \times 10^{8} \mathrm{Wcm}^{-3}$ for bulk GaAs and $\mathrm{AlGaAs} / \mathrm{GaAs}$ multi quantum wells. In parallel, the saturation index was of the order of $\delta n_{s} \simeq 2-3 \times 10^{-2}$. These comparisons are only qualitative and would require a specific study including the dot density as one of the parameters where the techniques reported here may certainly be pursued.

\section{Summary and conclusion}

In this chapter, we have discussed in detail the cw nonlinear optics of QD ensembles at room temperature. The model developed considers the important coupling between the QD states and the WL and the inhomogeneous broadening in determining the carrier populations in the dot in dependence on the incident light field. However, only the dielectric susceptibility of the GS is taken into account to calculate the back action (absorption and refractive index) of the QD medium back onto the light. We find saturation behavior of the absorption and gain in between the ones expected for purely homogeneously or purely inhomogeneously broadened media reflecting the complex intermediate situation in typical QD. Experiments on InAs and InAlAs QD yield the first demonstration of the saturation of absorption and thus open up the prospect of cw room-temperature nonlinear optics of QD. The satura- 
tion intensities inferred are about $5 \times 10^{8} \mathrm{~W} / \mathrm{m}^{2}$ for the InAs QD and $0.3 \times 10^{8} \mathrm{~W} / \mathrm{m}^{2}$ for the InAlAs QD. A detailed comparison between the values and with the theory is not only difficult because of the different nature of multi-pass effects involved in the measurements, but also because many of the other parameters involved are quite uncertain (e.g. defect density determining non-radiative lifetimes, the homogeneous broadening parameter $\gamma_{p}$ ) but the lower value observed for the InAlAs QD might indicate a better sample quality and less interaction with the semiconductor matrix due to the fact that there is only one well confined state. These uncertainties also make it difficult to compare the values obtained for the QD quantitatively to saturation intensities in bulk and quantum well samples. Values reported for good quality samples (i.e. lifetimes in the ns range) center around $(2-8) \times 10^{7} \mathrm{~W} / \mathrm{m}^{2}$ $[76,77,78]$, but can reach $4 \times 10^{8} \mathrm{~W} / \mathrm{m}^{2}$ [79] whereas the excitonic contribution (lifetime of $20 \mathrm{~ns}$ ) saturates already at $5.8 \times 10^{6} \mathrm{~W} / \mathrm{m}^{2}$ in the same sample [79]. We mentioned already that [74] reported a saturation intensity of $1-3 \times 10^{5} \mathrm{~W} / \mathrm{cm}^{2}$ for detuned excitation in the band tail of $\mathrm{Ga} / \mathrm{AlGaAs} \mathrm{QW}$.

The modal absorption was determined to be about $14 / \mathrm{cm}$ for the InAlAs QD (material absorption about $120 / \mathrm{cm}$ ) and about $14.5 / \mathrm{cm}$ (material absorption about $150 / \mathrm{cm}$ ) for the InAs QD. The latter value is somewhat smaller than the estimated one of $27 / \mathrm{cm}$. The maximum (modal) refractive index shift in edge-emitting samples is estimated to be on the order of $10^{-4}$, up to a few times $10^{-4}$, depending on the number of layers used. This should create a detectable phase shift in a nonlinear Fabry-Perot of a mm length or so, but preliminary corresponding experiments were unsuccessful in detecting these phase shifts, possibly the nonlinear interaction is reduced as indicated by the lower than expected values for the absorption.

For a clarification on the saturation behavior, future work should access the saturation properties of AR-coated or tilted samples such that the saturation is not influenced by multi-pass effects. Lifetime measurements need to be done on the very same samples in order to enable a more detailed comparison with the theoretical expectation. This can be expected to shed also some light on the influence of the coupling between WL (and ES) and GS states.

In order to promote devices for bistability, all-optical processing and solitons a high QD density, i.e. a high cooperativity parameter, and a low inhomogenous broadening are important, as well as a low defect density. For the point of growth, these are partially conflicting aims and one needs to aim for a good compromise. State-of-the-art InAs QD devices as investigated in this chapter have a density of $N_{Q D} \approx 5 \times 10^{10} \mathrm{~cm}^{-2}$ and an inhomogeneous broadening of about $40 \mathrm{~nm}$ or $30 \mathrm{meV}$. Estimations indicate that in an edge-emitting device with 10 QD layers a cooperativity parameter of about $C \approx 20$, i.e. in the regime where bistability might be accessible, can be reached in a cavity with $L=1.5 \mathrm{~mm}$ provided the Finesse is high enough $\left(R_{1}=R_{2}=0.99\right)$. A VCSEL with 20 QD layers could reach $C \approx 10$. Recent papers $[80,81]$ report $N_{Q D} \approx 5.9 \times 10^{10} \mathrm{~cm}^{-2}, 8$ layers and an inhomogeneous broadening of about $24 \mathrm{meV}$ giving $C \approx 30$ for an edge-emitting cavity with the parameters given above. Similarly, Ref. [82] reports $N_{Q D} \approx 8 \times 10^{10} \mathrm{~cm}^{-2}, 3$ layers and an inhomogeneous broadening of about $24 \mathrm{meV}$. Hence it appears that optimized SK-growth can reach interesting nonlinear regimes, but it should be cau- 
tioned that for SK-growth inhomogenous broadening is intrinsic. The broadening can be reduced by seeding techniques (e.g. $21 \mathrm{meV}$ [83], $17.5 \mathrm{meV}$ [84]), but currently only at a density of $N_{Q D} \approx 10^{10} \mathrm{~cm}^{-2}$. Alternative growth techniques like site-controlled growth [85, 86] yield inhomogeneous broadening as low as $4 \mathrm{meV}$ [85], but no QD density is given. Sub-monolayer QD are another promising material system with high gain and low broadening [36]. It should be noted that the single sheet density of $N_{Q D} \approx 2 \times 10^{11} \mathrm{~cm}^{-2}$ reported for InAlAs QD in Sec. 4 is probably close to the highest one achievable in a single layer before QD coalesce and optical properties degrade. This is in line with the fact that very high density samples with $N_{Q D} \approx 7.8 \times 10^{11} \mathrm{~cm}^{-2}$ were reported in [87], but apparently no devices were realized, yet.

In summary, it is hence our belief that devices based on staggered growth of optimized SK-layers or other advanced growth technologies can reach interesting nonlinear regimes and that the numerical and experimental investigations discussed in this chapter open up the prospect of cw nonlinear optics of QD, especially in the absorptive regime. Obviously, many details on the influence of inhomogeneous broadening and the influence of higher discrete and continuous states need to be worked out and explored, especially in view of devices relying on nonlinear phase shifts.

Acknowledgements T.A. and A.T. were supported by EPSRC project EP/E025021, A.T. gratefully acknowledges current funding of Région Provence Alpes Cote d'Azur (DEB 10-924) and a travel grant from COST action MP0702. M.B. acknowledges partial support from project FIRB PhoCOS project $n$. RBFR08QIP5. We are grateful to Innolume GmbH for supplying the InAs devices, J.-M. Benoit, A. Lemaittre, G. Patriarche, and K. Meunier for supplying the $780 \mathrm{~nm}$ devices, T. Maggipinto for supplying part of the numerical code, and R. Martin for computational support. S. Moore and T. Krauss were supplying broad-area samples for preliminary studies, and E. Esposito and G. McConnel gave us the opportunity to perform preliminary measurements in their lab.

\section{References}

1. B. Alèn, K. Karrai, R.J. Warburton, F. Bickel, P.M. Petro, J. Martínez-Pastord, Physica E 21, 395 (2004)

2. M.H. Baier, E. Pelucchi, E. Kapon, S. Varoutsis, M. Gallart, I. Robert-Philip, I. Abram, Appl. Phys. Lett. 84, 648 (2004)

3. D.J. Mowbray, M.S. Skolnick, J. Phys. D: Appl Phys. 38, 2059 (2005)

4. S. Schneider, P. Borri, W. Langbein, U. Woggon, J. Förstner, A. Knorr, R.L. Sellin, D. Ouyang, D. Bimberg, Appl. Phys. Lett. 83, 3668 (2003)

5. Y. Sugiyama, Y. Nakata, S. Muto, T. Futatsugi, N. Yokoyama, IEEE Sel. Top. Quantum Electron. 4, 880 (1998)

6. T. Akiyama, H. Kuwatsuka, T. Simoyama, Y. Nakata, K. Mukai, M. Sugawara, O. Wada, H. Ishikawa, Opt. Quantum Electron. 33, 927 (2001)

7. H. Nakamura, K. Kanamoto, Y. Nakamura, S. Ohkouchi, H. Ishikawa, K. Asakawa, J. Appl. Phys. 96, 1425 (2004)

8. K.W. Su, H.C. Lai, A. Li, Y.F. Chen, K.F. Huang, Opt. Lett. 30, 1482 (2005) 
9. V. Cesari, W. Langbein, P. Borri, M. Rossetti, A. Fiore, S. Mikhrin, I. Krestnikov, A. Kovsh, Appl. Phys. Lett. 90, 201103 (2007)

10. S. Dommers, V.V. Temnov, U. Woggon, J. Gomis, J. Martinez-Pastor, M. Laemmlin, D. Bimberg, Appl. Phys. Lett. 90, 033508 (2007)

11. I. OâDriscoll, T. Piwonski, J. Houlihan, G. Huyet, R.J. Manning, B. Corbett, Appl. Phys. Lett. 91, 263606 (2007)

12. J. Pulka, T. Piwonski, G. Huyet, J. Houlihan, S. Barbay, A. Martinez, K. Merghem, A. Lemaître, A. Ramdane, R. Kuszelewicz, IEEE J. Quantum Electron. (2011). To appear.

13. T.W. Berg, J. Mørk, J.M. Hvam, New J. Phys. 6, 178 (2004)

14. M. Sugawara, H. Ebe, N. Hatori, M. Ishida, Y. Arakawa, T. Akiyama, K. Otsubo, Y. Nakata, Phys. Rev. B 69, 235332 (2004)

15. A.V. Uskov, E. O’Reilly, M. Laemmlin, N.N. Ledentsov, D. Bimberg, Opt. Commun. 248, 211 (2005)

16. C. Meuer, K. Kim, M. Laemmlin, S. Liebich, A. Capua, G. Eisenstein, A.R. Kovsh, S.S. Mikhrin, I.L. Krestnikov, D. Bimberg, Opt. Exp. 16, 8269 (2008)

17. D.J.H.C. Maas, A.R. Bellancourt, M. Hoffmann, B. Rudin, Y. Barbarin, M. Golling, T. Südmeyer, U. Keller, Opt. Exp. 16, 18646 (2008)

18. M.P. Lumb, P.N. Stavrinou, E.M. Clarke, R. Murray, C.G. Leburn, C. Jappy, N.K. Metzger, C.T.A. Brown, W. Sibbett, Appl. Phys. B 97, 53 (2009)

19. T. Piwonski, J. Pulka, G. Madden, G. Huyet, J. Houlihan, E.A. Viktorov, T. Erneux, P. Mandel, Appl. Phys. Lett. 94, 123504 (2009)

20. F. Wu, W. Tian, W. Chen, G. Zhang, G. Zhao, S. Cao, W. Xie, J. Mod. Opt. 56, 1868 â1873 (2009)

21. I. Dancus, V.I. Vlad, A. Petris, N. Gaponik, V. Lesnyak, A. Eychmüller, Opt. Lett. 35, 1079 (2010)

22. S. Barbay, J. Koehler, R. Kuszelewicz, T. Maggipinto, I.M. Perrini, M. Brambilla, IEEE J. Quantum Electron. 39, 245 (2003)

23. I.M. Perrini, S. Barbay, T. Maggipinto, M. Brambilla, R. Kuszelewicz, Appl. Phys. B 81, 905 (2005)

24. M. Brambilla, T. Maggipinto, I.M. Perrini, S. Barbay, R. Kuszelewicz, Chaos 17, 037119 (2007)

25. A. Tierno, T. Ackemann, T. Maggipinto, M. Brambilla, Phys. Rev. B 80, 035314 (2009)

26. H. Su, S. Chuang, Opt. Lett. 31, 271 (2006)

27. N. Akhmediev, A. Ankiewicz (eds.), Dissipative solitons, Lecture Notes in Physics, vol. 661 (Springer, Berlin, 2005)

28. T. Ackemann, G.L. Oppo, W.J. Firth, Adv. Atom. Mol. Opt. Phys. 57, 323 (2009)

29. R. Kuszelewicz, S. Barbay, G. Tissoni, G. Almuneau, Eur. Phys. J. D 59, 1 (2010)

30. S. Barland, J.R. Tredicce, M. Brambilla, L.A. Lugiato, S. Balle, M. Giudici, T. Maggipinto, L. Spinelli, G. Tissoni, T. Knödel, M. Miller, R. Jäger, Nature 419, 699 (2002)

31. T. Ackemann, W.J. Firth, in Dissipative solitons, Lecture Notes in Physics, vol. 661, ed. by N. Akhmediev, A. Ankiewicz (Springer, Berlin, 2005), pp. 55-100

32. F. Pedaci, S. Barland, E. Caboche, P. Genevet, M. Giudici, J.R. Tredicce, T. Ackemann, A.J. Scroggie, W.J. Firth, G.L. Oppo, G. Tissoni, R. Jäger, Appl. Phys. Lett. 92, 011101 (2008)

33. V.B. Taranenko, G. Slekys, C. Weiss, in Dissipative Solitons, ed. by N. Akhmediev, A. Ankiewicz, Lecture Notes in Physics (Springer, New York, 2005), pp. 131-160

34. C.H. Henry, IEEE J. Quantum Electron. 18, 259 (1982)

35. P.M. Smowton, E.J. Pearce, H.C. Schneider, W.W. Chow, M. Hopkinson, Appl. Phys. Lett. 81, $3251(2002)$

36. Z. Xu, D. Birkedal, M. Juhl, J.M. Hvam, Appl. Phys. Lett. 85, 3259 (2004)

37. C. Ribbat, R.L. Selin, I. Kaiander, F. Hopfer, N.N. Ledentsov, D. Bimberg, A.R. Kovsh, V.M. Ustinov, A.E. Zhukov, M.V. Maximov, Appl. Phys. Lett. 82, 952 (2003)

38. S. Schneider, P. Borri, W. Langbein, U. Woggon, R.L. Sellin, D. Ouyang, D. Bimberg, IEEE J. Quantum Electron. 40, 1423 (2004)

39. S. Melnik, G. Huyet, A.V. Uskov, Opt. Exp. 14, 2950 (2006) 
40. S.P. Hegarty, B. Corbett, J.G. McInerney, G. Huyet, Electron. Lett. 41, 416 (2005)

41. A. Tierno, T. Ackemann, C.G. Leburn, C.T.A. Brown, Appl. Phys. Lett. 97, 23231104 (2010)

42. R. Kuszelewicz, J.M. Benoit, S. Barbay, A. Lemaitre, G. Patriarche, K. Meunier, A. Tierno, T. Ackemann, (2011). Subm. to J. Appl. Phys.

43. M. Paillard, X. Marie, P. Renucci, T. Amand, A. Jbeli, J.M. Gérard, Phys. Rev. Lett. 86, 1634 (2001)

44. E. Tsitsishvili, R.v. Baltz, H. Kalt, Phys. Rev. B 66, 161405(R) (2002)

45. A.V. Uskov, Y. Boucher, J. Le Bihan, J. McInerney, Appl. Phys. Lett. 73, 1499 (1998)

46. A. Markus, J.X. Chen, O. Gauthier-Lafaye, J.G. Provost, C. Paranthoen, A. Fiore, IEEE Sel. Top. Quantum Electron. 9, 1308 (2003)

47. E.A. Viktorov, P. Mandel, Y. Tanguy, J. Houlihan, G. Huyet, Appl. Phys. Lett. 87, 053113 (2005)

48. M. Brambilla, L.A. Lugiato, F. Prati, L. Spinelli, W.J. Firth, Phys. Rev. Lett. 79, 2042 (1997)

49. L. Spinelli, G. Tissoni, M. Brambilla, F. Prati, L.A. Lugiato, Phys. Rev. A 58, 2542 (1998)

50. A.R. Kovsh, N.A. Maleev, A.E. Zhukov, S.S. Mikhrin, A.P. Vasilâev, Y.M. Shernyakov, M.V. Maximov, D.A. Livshits, V.M. Ustinov, Z.I. Alferov, N.N. Ledentsov, D. Bimberg, Electron. Lett. 38, 1104 (2002)

51. A.E. Zhukov, A.R. Kovsh, S.S. Mikhrin, A.P. Vasil'ev, E.S. Semenova, N.A. Maleev, V.M. Ustinov, M.M. Kulagina, E.V. Nikitina, I.P. Soshnikov, Y.M. Shernyakov, D.A. Livshits, N.V. Kryjapovskykaya, D.S. Sizov, M.V. Maximov, A.F. Tsatsul'nikov, N.N. Ledentsov, B. D., Z.I. Alferov, Physica E 17, 589 (2003)

52. P. Borri, W. Langbein, J. Mørk, J.M. Hvam, F. Heinrichsdorff, M.H. Mao, D. Bimberg, Phys. Rev. B 60, 7784 (2004)

53. A. Yariv, Quantum Electronics, 3rd edn. (John Wiley \& Sons, New York, 1988)

54. M. Sugawara, K. Mukai, Y. Nakata, Appl. Phys. Lett. 74, 1561 (1999)

55. A. Capua, V. Mikhelashvili, G. Eisenstein, J.P. Reithmaier, A. Somers, A. Forchel, M. Calligaro, O. Parillaud, M. Krakowski, Opt. Exp. pp. 2141-2146 (2008)

56. L. Thylén, Opt. Quantum Electron. 15, 433 (1983)

57. M. Sheik-Bahae, A.A. Said, T.H. Wei, D.J. Hagan, E.W.V. Stryland, IEEE J. Quantum Electron. 26, 760 (1990)

58. A.E. Siegman, Lasers (University Science Books, Mill Valley, California, 1986)

59. T. Ackemann, T. Scholz, C. Vorgerd, J. Nalik, L.M. Hoffer, G.L. Lippi, Opt. Commun. 147, 411 (1998)

60. G. Labeyrie, T. Ackemann, B. Klappauf, M. Pesch, G.L. Lippi, R. Kaiser, Eur. Phys. J. D 22, $473(2003)$

61. Y. Tanguy, T. Ackemann, W.J. Firth, R. Jäger, Phys. Rev. Lett. 100, 013907 (2008)

62. M. Gioannini, A. Sevega, V. Montrosset, Opt. Quant. Electron. 38, 381 (2006)

63. G. Tissoni, L. Spinelli, L.A. Lugiato, M. Brambilla, Proc. SPIE 4283, 577 (2001)

64. H.C. Schneider, W.W. Chow, S.W. Koch, Phys. Rev. B 64, 115315 (2001)

65. H.C. Schneider, W.W. Chow, S.W. Koch, Phys. Rev. B 66, 041310(R) (2002)

66. G. Huyet (2005). Personal Communication.

67. H.C. Wong, G.B. Ren, J.M. Rorison, Opt. Quant. Electron. 38, 395 (2006)

68. A.A. Lagatsky, C.G. Leburn, C.T.A. Brown, W. Sibbett, S. Zolotovskaya, E.U. Rafailov, Progr. Quant. Electron. 34, 1 (2010)

69. J.Y. Marzin, J.M. Gérard, A. Izraël, D. Barrier, Phys. Rev. Lett. 94, 716 (1994)

70. R. Leon, S. Fafard, D. Leonard, J.L. Merz, P.M. Petroff, Appl. Phys. Lett. 67, 521 (1995)

71. K. Hinzer, P. Hawrylak, M. Korkusinski, S. Fafard, M. Bayer, O. Stern, A. Gorbunov, A. Forchel, Phys. Rev. B 63, 075314 (2001)

72. D.A.B. Miller, IEEE J. Quantum Electron. 17, 306 (1981)

73. B.S. Wherrett, IEEE J. Quantum Electron. 20, 646 (1984)

74. B.G. Sfez, J.L. Oudar, R. Kuszelewicz, D. Pellat, Appl. Phys. Lett. 80, 1163 (1992)

75. S. Adachi, J. Appl. Phys. 58(3), R1 (1985)

76. J.S. Weiner, D.B. Pearson, D.A.B. Miller, D.S. Chemla, D. Sivco, A.Y. Cho, Appl. Phys. Lett. 49, 531 (1986) 
77. Y.H. Lee, A. Chavez-Pirson, S.W. Koch, H.M. Gibbs, S.H. Park, J. Morhange, A. Jeffery, N. Peyghambarian, L. Banyai, A.C. Gossard, W. Wiegmann, Phys. Rev. Lett. 57, 2446 (1986)

78. C. Tombling, T. Saitoh, Y. Suzuki, H. Tanaka, Electron. Lett. 27, 1374 (1991)

79. D.A.B. Miller, D.S. Chemla, D.J. Eilenberger, P.W. Smith, A.C. Gossard, W.T. Tsang, Appl. Phys. Lett. 41, 679 (1983)

80. K. Takada, Y. Tanaka, T. Matsumoto, M. Ekawa, H.Z. Song, Y. Nakata, M. Yamaguchi, K. Nishi, T. Yamamoto, M. Sugawara, Y. Arakawa, Electron. Lett. 47, 206 (2011)

81. T. Kageyama, K. Nishi, M. Yamaguchi, R. Mochida, Y. Maeda, K. Takemasa, T. Yamamoto, M. Sugawara, Y. Arakawa. Extremely High Temperature $\left(220^{\circ} \mathrm{C}\right)$ Continuous-Wave Operation of 1300-nm-range Quantum-Dot Lasers. CLEO-Europe, Munich, 2011. Paper PDA.1-Tue (2011)

82. T. Amano, T. Sugaya, K. Komori, IEEE Photon. Tech. Lett. 18, 619 (2006)

83. K. Nishi, H. Saito, S. Sugou, J.S. Lee, Appl. Phys. Lett. 74, 1111 (1999)

84. Z. Mi, P. Bhattacharyaa, J. Appl. Phys. 98, 023510 (2005)

85. K. Leifer, E. Pelucchi, S. Watanabe, F. Michelini, B. Dwir, E. Kapon, Appl. Phys. Lett. 91, 081106 (2007)

86. N. Gogneau, L. Le Gratiet, E. Cambril, G. Beaudoin, G. Patriarche, A. Beveratos, R. Hostein, I. Robert-Philip, J.Y. Marzin, I. Sagnes, J. Cryst. Growth 310, 3413 (2008)

87. C.K. Chia, Y.W. Zhang, S.S. Wong, A.M. Yong, S.Y. Chow, S.J. Chua, J. Guo, Appl. Phys. Lett. 90, 161906 (2007) 